\title{
Synthesis, Characterization, and Biological Evaluation of Some New Functionalized Terphenyl Derivatives
}

\author{
Seranthimata Samshuddin, ${ }^{1}$ Badiadka Narayana, ${ }^{1}$ Balladka Kunhanna Sarojini, ${ }^{2}$ \\ Divya N. Shetty, ${ }^{1}$ and Nalilu Suchetha Kumari ${ }^{3}$ \\ ${ }^{1}$ Department of Studies in Chemistry, Mangalore University, Mangalagangotri, Karnataka, Mangalore 574 199, India \\ ${ }^{2}$ Research Department of Chemistry, P.A. College of Engineering, Nadupadavu, Karnataka, Mangalore 574153, India \\ ${ }^{3}$ Department of Biochemistry, K. S. Hegde Medical Academy, Deralakatte 574162, India
}

Correspondence should be addressed to Badiadka Narayana, nbadiadka@yahoo.co.uk

Received 23 August 2012; Revised 20 November 2012; Accepted 20 November 2012

Academic Editor: Armando Rossello

Copyright (c) 2012 Seranthimata Samshuddin et al. This is an open access article distributed under the Creative Commons Attribution License, which permits unrestricted use, distribution, and reproduction in any medium, provided the original work is properly cited.

\begin{abstract}
New functionalized terphenyl derivatives incorporating various heterocyclic rings are prepared by using $4,4^{\prime \prime}$-difluoro- $5^{\prime}$-hydroxy$1,1^{\prime}: 3^{\prime}, 1^{\prime \prime}$-terphenyl-4' -carbohydrazide as a key intermediate derived from $4,4^{\prime}$-difluoro chalcone, a versatile synthone. All the derivatives are characterized by ${ }^{1} \mathrm{H}$ NMR, IR, and mass spectral data. All the synthesized products are screened for their in vitro antimicrobial and antioxidant properties. The majority of the tested compounds exhibited significant antioxidant activity and some of them showed good antimicrobial activity.
\end{abstract}

\section{Introduction}

Terphenyl is a common structural motif found in various natural products, largely isolated from microbes and mushrooms [1]. In recent years, it has been reported that some terphenyls exhibit considerable biological activities, for example, potent anticoagulants, immunosuppressants, antithrombotic, neuroprotective, specific 5-lipoxygenase inhibitory, and cytotoxic activities [2]. Because of their promising biological activities and important properties, terphenyls have been produced increasing research interest. Therefore, synthesis of terphenyl derivatives has been a fascinating area in organic field [3].

Recently we have reported a new and simple method for the preparation of ester derivative of terphenyl starting from $4,4^{\prime}$-difluoro chalcone [4]. The esters can be easily converted into the corresponding carbohydrazide by the reaction with hydrazine hydrate. Hydrazides and related compounds have been described as useful precursors for the assembly of various heterocyclic rings. A large number of hydrazides are reported to be of biological interest $[5,6]$, while oxadiazole derivatives and thiosemicarbazides have been reported to possess antibacterial $[7,8]$, antifungal $[9,10]$, and other biological activities. Furthermore, a number of substituted thiazolines and thiazolidinones are found to exhibit appreciable antimicrobial activities [11-15].

In the course of investigation, we have found that $4,4^{\prime \prime}$ difluoro- $5^{\prime}$-hydroxy-1, $1^{\prime}: 3^{\prime}, 1^{\prime \prime}$-terphenyl- $4^{\prime}$-carbohydrazide is an excellent building block for the synthesis of numerous heterocyclic systems. In view of the pharmacological importance of terphenyls and in continuation of our work on synthesis of various derivatives of 4,4' -difluoro chalcone [1623 ], it was decided to prepare new functionalized terphenyl derivatives by using $4,4^{\prime \prime}$-difluoro- $5^{\prime}$-hydroxy-1, $1^{\prime}: 3^{\prime}, 1^{\prime \prime}$ terphenyl- $4^{\prime}$-carbohydrazide as the key intermediate and to study their biological activities.

\section{Chemistry}

A novel terphenyl derivative, ethyl 4,4" $4^{\prime \prime}$ difluoro-5' -hydroxy$1,1^{\prime}: 3^{\prime}, 1^{\prime \prime}$-terphenyl- $4^{\prime}$-carboxylate 3 , was synthesized by the oxidative aromatization of cyclohexenone derivative of 4, $4^{\prime}$ difluoro chalcone using chloramine-T [4]. The ester 3 was converted into the corresponding carbohydrazide 4 in high yield by the reaction with hydrazine hydrate (Scheme 1). In the IR spectrum of carbohydrazide 4 broad stretching 


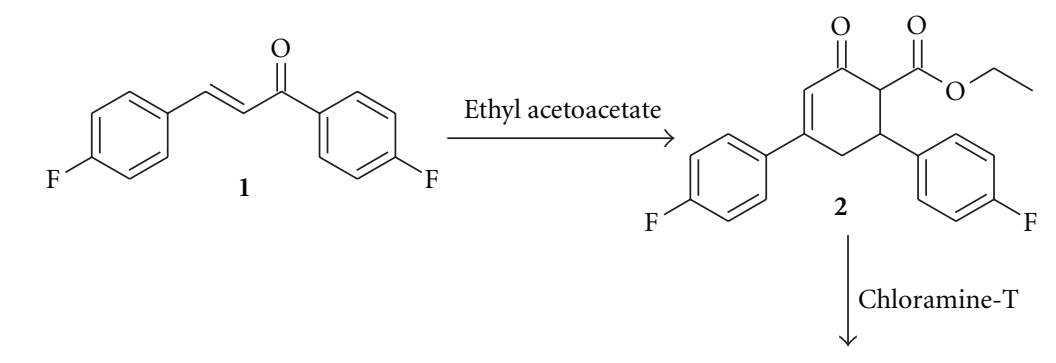<smiles>NNC(=O)c1c(O)cc(-c2ccc(F)cc2)cc1-c1ccc(F)cc1</smiles><smiles>CCOC(=O)c1c(O)cc(-c2ccc(F)cc2)cc1-c1ccc(F)cc1</smiles>

SCHEMe 1

band at $3290 \mathrm{~cm}^{-1}$ was due to $\mathrm{NH}$ while strong stretching band at $1672 \mathrm{~cm}^{-1}$ was attributed to carbonyl. ${ }^{1} \mathrm{H}$ NMR spectrum showed two singlets at $\delta 9.19 \mathrm{ppm}$ and $\delta 4.18 \mathrm{ppm}$ which were accounted for $\mathrm{NH}_{2}$ and $\mathrm{NH}$ protons. The mass spectrum of 4 showed a molecular ion peak at $\mathrm{m} / z 341.0$ which confirmed its molecular weight.

The carbohydrazide is a versatile key intermediate for the synthesis of several heterocyclic systems such as pyrazoles, thiazoles, 1,3,4-oxadiazoles, and 1,2,4-triazoles [24, 25]. Hence, the carbohydrazide 4 was treated with various reagents to afford pyrazole and 1,3,4-oxadiazole derivatives (Scheme 2). Condensation reaction of carbohydrazide 4 with dicarbonyl compounds, namely, acetylacetone and ethyl acetoacetate, in the presence of catalytic amount of triethylamine afforded the corresponding substituted pyrazole derivatives $\mathbf{5}$ and $\mathbf{6}$, respectively. The IR spectrum of pyrazole 5 showed the presence of vibrations at $2926 \mathrm{~cm}^{-1}$ due to methyl group. The ${ }^{1} \mathrm{H}$ NMR spectrum of $\mathbf{5}$ showed two singlets at $\delta 2.29$ and $2.70 \mathrm{ppm}$ due to three each protons of two methyl groups at C-3 and C-5 carbon of pyrazole ring, respectively, and hence confirming the presence of 3,5dimethyl-1H-pyrazole moiety. Similarly, in the IR spectrum of pyrazole 6, vibrations at $1654 \mathrm{~cm}^{-1}$ indicates the presence of carbonyl group of the pyrazolone nuclei. Singlets at $\delta 2.84$ and $2.03 \mathrm{ppm}$ in the ${ }^{1} \mathrm{H}$ NMR spectrum were observed for the methyl and methylene protons of the pyrazolone moiety.

Cyclization to the oxadiazoles was carried out by treating the carbohydrazide 4 with triethyl orthoformate/toluene, cyanogen bromide/ethanol and with aromatic acids/phosphorous oxychloride. The IR spectra of oxadiazoles 7 and 9 showed the absence of vibrations due to $\mathrm{NH}$ and $\mathrm{NH}_{2}$ groups. Their ${ }^{1} \mathrm{H}$ NMR spectra also indicated the absence of $\mathrm{NH}$ protons, thus confirmed the cyclization reaction. In the IR spectrum of oxadiazole 8 , a stretching vibration observed at $3385 \mathrm{~cm}^{-1}$ which indicates the presence of amino group. A singlet at $\delta 6.93 \mathrm{ppm}$ in the ${ }^{1} \mathrm{H}$ NMR spectrum of 8 confirmed the presence of $\mathrm{NH}_{2}$ group and hence proved that ring closure starting from carbohydrazide 4 resulted in the formation of 5-amino-1,3,4-oxadiazole moiety.
An arylidine hydrazone incorporated into the parent terphenyl was also synthesized. Thus, condensation of carbohydrazide 4 with aromatic aldehydes in absolute ethanol in presence of few drops of concentrated hydrochloric acid afforded the corresponding Schiff bases 10a-c. The Schiff bases 10a-c thus obtained were cyclized into 1,3,4oxadiazole derivatives $11 \mathrm{a}-\mathrm{c}$ by refluxing them with acetic anhydride (Scheme 3). IR spectra of $\mathbf{1 0 a}-\mathbf{c}$, showed an $\mathrm{NH}$ stretching band at around $3329 \mathrm{~cm}^{-1}$ and a carbonyl absorption band near $1647 \mathrm{~cm}^{-1}$. The ${ }^{1} \mathrm{H}$ NMR spectrum of $10 \mathrm{c}$, as an example, showed a singlet signal at $\delta 8.15 \mathrm{ppm}$ corresponding to $\mathrm{CH}$ of the benzylidene group and another singlet signal at $\delta 11.71 \mathrm{ppm}$ due to $\mathrm{NH}$ proton, hence confirming the formation of Schiff base hydrazone. The IR spectra of compounds 11a-c had different characteristics since they showed no N-H stretching bands, but carbonyl stretching near $1770 \mathrm{~cm}^{-1}$ which could be attributed to the carbonyl of acetyl group. ${ }^{1} \mathrm{H}$ NMR spectra of $11 \mathbf{a}-\mathbf{c}$ showed a singlet in the spectra in the range $\delta 6.41-6.52 \mathrm{ppm}$ indicating $\mathrm{CH}$ resonance of the oxadiazoline ring which is in accordance with the reported values found in the literature [26].

The treatment of hydrazides with carbon disulfide in the presence of alkali hydroxide was a general method leading to the formation of 1,3,4-oxadiazole-2-thione derivatives [27]. As the starting material, carbohydrazide 4 was used for the synthesis of 1,3,4-oxadiazole-2-thione derivative $\mathbf{1 2}$ (Scheme 4). The IR spectrum of compound 12 showed a sharp absorption band at $3224 \mathrm{~cm}^{-1}$ representing $\mathrm{NH}$ group. Its ${ }^{1} \mathrm{H}$ NMR spectrum displayed a signal at $\delta 14.53 \mathrm{ppm}$ due to the $\mathrm{NH}$ proton, while no signal representing a hydrazide structure appeared. The 4-amino-1,2,4-triazole3 -thione derivative 13 was synthesized starting from compound 12 by treating it with hydrazine hydrate. The IR spectrum of compound $\mathbf{1 3}$ showed two sharp absorption bands at $3373 \mathrm{~cm}^{-1}$ and $3259 \mathrm{~cm}^{-1}$ representing $-\mathrm{NH}$ and $\mathrm{NH}_{2}$ groups. The singlet signal integrating two protons seen at $\delta 5.45 \mathrm{ppm}$ in the ${ }^{1} \mathrm{H}$ NMR spectrum of compound 13 was attributed to the $-\mathrm{NH}_{2}$ group. Furthermore, an additional 


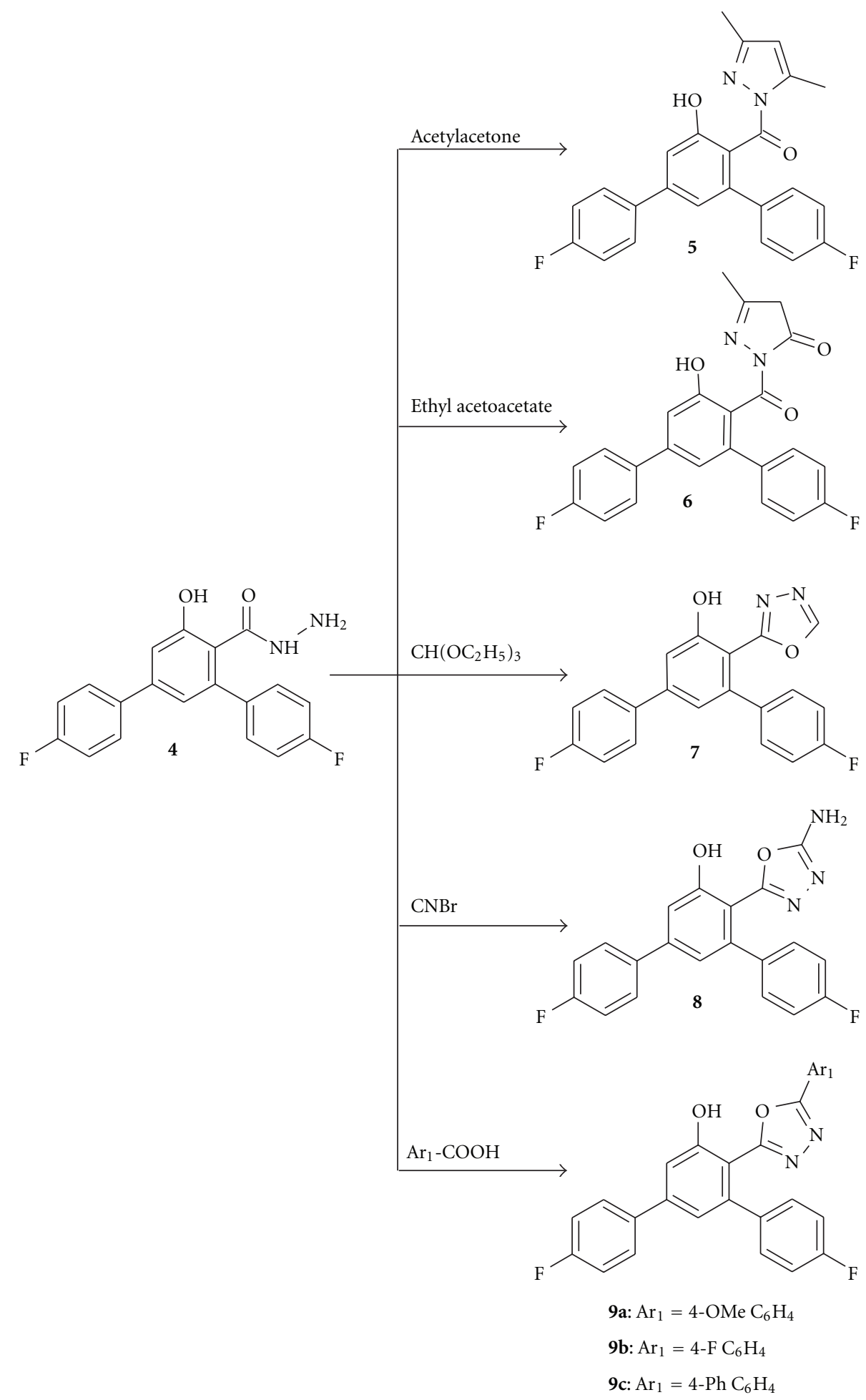

SCheme 2

singlet was observed due to the $-\mathrm{NH}$ group at $\delta 13.54 \mathrm{ppm}$ integrating one proton.

Mannich reaction of 1,3,4-oxadiazole-2-thione derivative 12 with secondary amines and formaldehyde in absolute ethanol at room temperature yielded the desired compounds 14a-c. When compared with the compound 12, the IR spectra of Mannich bases 14a-c showed the absence of $\mathrm{NH}$ group. Their ${ }^{1} \mathrm{H}$ NMR spectra also supported the proposed structure as there was no signal corresponding to $\mathrm{NH}$ proton. Further, 1,3,4-oxadiazole-2-thione derivative $\mathbf{1 2}$ was 


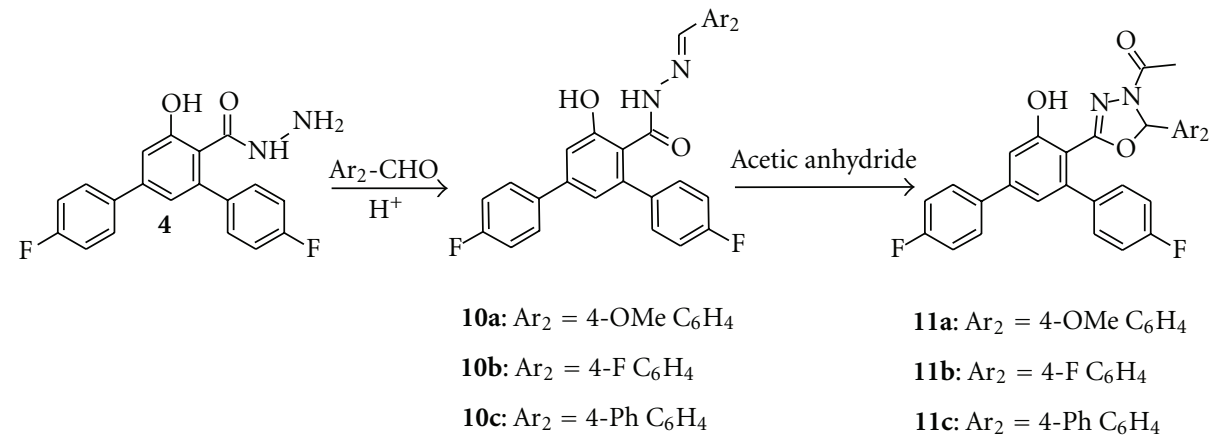

Scheme 3

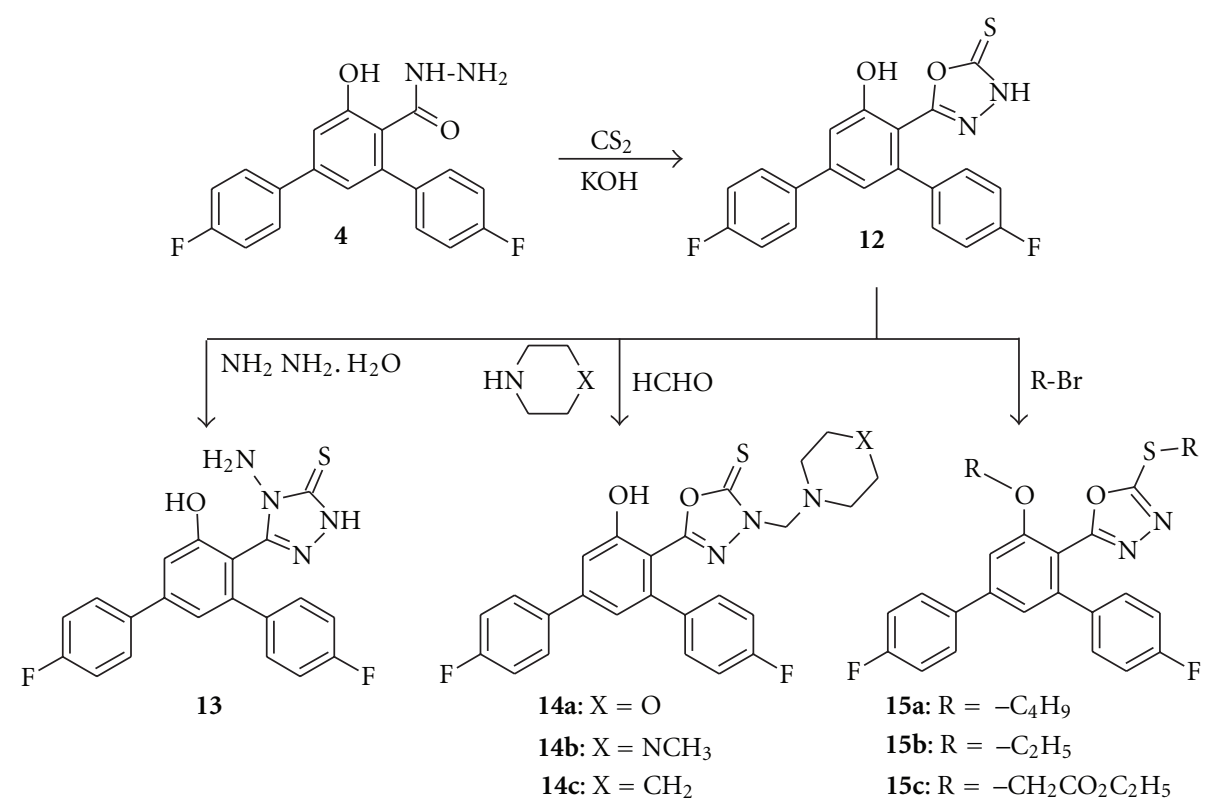

SCHEME 4

alkylated using different alkyl halides. Compound $\mathbf{1 2}$ can exist in thione or thiol tautomeric forms. However, it has been established that under the action of alkyl halides $S$ substitution took place instead of $N$-substitution. The mass spectrum of $15 \mathrm{c}$ showed a molecular ion peak at $\mathrm{m} / z 555.1$ which reveals that the compound not only $S$-alkylated but also $O$-alkylated, because of the presence of phenolic group.

Furthermore, the intermediate thiosemicarbazide derivative 16 representing a versatile building block for the synthesis of new heterocycles incorporating thiazole, imidazole, and triazole nucleus was synthesized by heating the carbohydrazide 4 with potassium thiocyanate in the presence of hydrochloric acid $[28,29]$ (Scheme 5). The ${ }^{1} \mathrm{H}$ NMR spectrum of 16 exhibited singlet signals at $\delta 6.96 \mathrm{ppm}$ and $8.19 \mathrm{ppm}$ due to $\mathrm{NH}_{2}$ protons as one of the proton involved in intramolecular hydrogen bonding with carbonyl group; two protons were unequal. In addition to that, it showed two singlets at $\delta 9.57$ and $10.45 \mathrm{ppm}$ due to two $\mathrm{NH}$ protons. The IR spectrum of 16 exhibited two sharp absorption bands at $3464 \mathrm{~cm}^{-1}$ and $3329 \mathrm{~cm}^{-1}$ representing
$-\mathrm{NH}$ and $-\mathrm{NH}_{2}$ groups. The thiazole derivatives $17 \mathbf{a}-\mathbf{c}$ was synthesized by the treatment of thiosemicarbazide derivative 16 with different phenacyl bromides in boiling ethanol. These reactions were assumed to proceed via $S$-alkylation followed by dehydration. The IR spectra of thiazoles $17 \mathbf{a}-$ c showed absorption band at $3331 \mathrm{~cm}^{-1}$ representing $-\mathrm{NH}$ groups. Its ${ }^{1} \mathrm{H}$ NMR spectra exhibited two singlets in the region $\delta$ 9.4-10.5 ppm for hydrazide $(\mathrm{NH})$ protons, and hence confirming the formation of thiazole moiety.

Compound 16 was cyclized via oxidative cyclization in basic medium $(10 \% \mathrm{NaOH})$, under reflux condition with subsequent acidification to give 1,2,4-triazole-3-thiol derivative 18. Further, reflux of compound $\mathbf{1 6}$ with monochloroacetic acid and anhydrous sodium acetate in glacial acetic acid afforded a product identified as 2-imino-4-oxo-1,3thiazolidine derivative 19 (Scheme 5). The structure of the synthesized products 18 and 19 was established on the basis of spectral data. For example, in compound 18, the absence of absorption band of carbonyl group in IR spectrum and lack of ${ }^{1} \mathrm{H}$ NMR resonances observed with $\mathrm{NH}$ and 
<smiles>NC(=O)NCNC(=O)c1c(O)cc(-c2ccc(F)cc2)cc1-c1ccc(F)cc1</smiles>

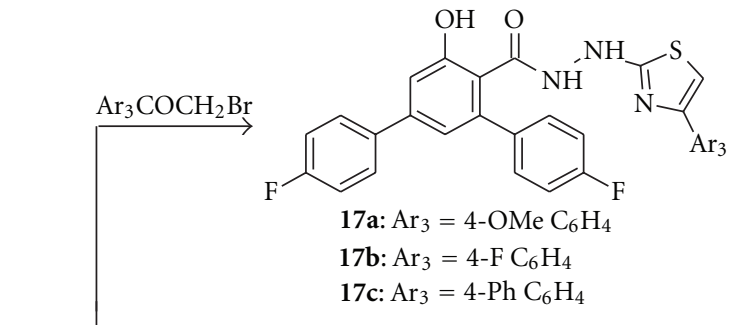<smiles>CC(O)C(C)C(=O)OCC(=O)O</smiles>

SCHEME 5

$\mathrm{NH}_{2}$ functions proved the intramolecular cyclization of compound 16 resulted in the formation of 1,2,4-triazole ring. Similarly, IR spectrum of thiazolidine derivative 19 showed an absorption band at $1724 \mathrm{~cm}^{-1}$ representing carbonyl group of imidazolidinone moiety. A singlet at $\delta 3.88 \mathrm{ppm}$ present in the ${ }^{1} \mathrm{H}$ NMR spectrum of 19 indicated the two protons of methylene group of imidazolidinone moiety, in addition to a singlet at $\delta 4.10 \mathrm{ppm}$ due to $\mathrm{SH}$ proton and another singlet at $\delta 10.59 \mathrm{ppm}$ due to that $\mathrm{NH}$ proton confirmed the presence of imidazolidinone moiety.

\section{Biological Evaluation}

3.1. Antibacterial Studies. The newly synthesized compounds were screened for their antibacterial activity against Escherichia coli (ATTC-25922), Staphylococcus aureus (ATTC25923), Pseudomonas aeruginosa (ATCC-27853), and Klebsiella pneumoniae (recultured) bacterial strains by serial plate dilution method [30, 31]. Ciprofloxacin was used as standard antibiotics for antibacterial activity.

Compound 12 was found to be the most active derivative against Escherichia coli, Pseudomonas aeruginosa, and Klebsiella pneumoniae at MIC value of $6.25 \mu \mathrm{g} / \mathrm{mL}$ among the tested compounds and showed the same potency with ciprofloxacin. The compounds $3,4,5,6,16,17 \mathrm{a}, 17 \mathrm{~b}$, and $17 \mathrm{c}$ were found to be active against all the tested microorganisms at MIC value of $12.5 \mu \mathrm{g} / \mathrm{mL}$. The high activity of 12 was due to the presence of 1,3,4-oxadiazole-2-thione moiety containing free $\mathrm{NH}$ group. Also the derivatives containing hydrazide moiety $(4,16,17 \mathbf{a}, 17 \mathbf{b}, 17 \mathrm{c})$, ester group (3), and pyrazole moiety ( $5 \& 6$ ) were helped to inhibit the growth of the microorganisms.
3.2. Antifungal Studies. Newly prepared compounds were screened for their antifungal activity against Aspergillus flavus (NCIM No. 524), Aspergillus fumigates (NCIM No. 902), Penicillium (S. aureus) (recultured), and Trichophyton mentagrophytes (recultured) in DMSO by serial plate dilution method [32, 33]. Activity of each compound was compared with itraconazole as standard.

Compounds $\mathbf{6}, \mathbf{8}$, and $\mathbf{1 0 b}$ were found to be the most active derivatives against all the tested microorganisms at an MIC value of $6.25 \mu \mathrm{g} / \mathrm{mL}$ among the tested compounds, and showed the same potency with itraconozole. Although compounds 9a, 9c, 10a, 11a, 11b, 11c, 17a, and 19 showed activity having MIC value of $12.5 \mu \mathrm{g} / \mathrm{mL}$ against selected microorganisms, they exhibited lower potencies than the compared standard drug. Hence, it could be concluded that different functionality present in the molecules contributed to the antifungal activity in different manner comparable with that of standard drug tested.

3.3. DPPH Radical Scavenging Assay. A rapid, simple, and inexpensive method to measure antioxidant capacity of substances involves the use of the free radical, 2,2-Diphenyl1-picrylhydrazyl (DPPH). DPPH is widely used to test the ability of compounds to act as free radical scavengers or hydrogen donors. Antioxidants tested on DPPH were also found extremely effective in cell systems. This simple test further provides information on the ability of a compound to donate electrons during antioxidant action [34]. The radical scavenging mechanism is based on the transfer of acidic $\mathrm{H}$ atom from the compound to DPPH radical to form DPPHH. 
Among the tested compounds, many compounds showed significant radical scavenging capacity due to the presence of phenolic hydrogen in the parent molecule. Compounds 12 and 13 showed good radical scavenging capacity while compounds 9a, 9b, 14a, 14b, 15c, and 16 exhibited moderate radical scavenging capacity with concentration of $1 \mathrm{mg} / \mathrm{mL}$ in comparison with the standard glutathione. The good radical scavenging capacity of compounds 12 and 13 was due to the presence of acidic protons in the oxadiazole moiety. The variation exhibited in DPPH scavenging capacity could be attributed to the effect of different moiety present in the compounds.

3.4. Reducing Power Assay. Reducing power is associated with antioxidant activity and may serve as a significant reflection of the antioxidant activity [35]. Compounds with reducing power indicate that they are electron donors and can reduce the oxidized intermediates of lipid peroxidation processes, so that they can act as primary and secondary antioxidants [36]. Substances, which have reduction potential, react with potassium ferricyanide $\left(\mathrm{Fe}^{3+}\right)$ to form potassium ferrocyanide $\left(\mathrm{Fe}^{2+}\right)$, which then reacts with ferric chloride to form ferrous complex that has an absorption maximum at $700 \mathrm{~nm}$. Increased absorbance of the reaction mixture indicates the increased reducing power.

Reducing power assay is expressed in effective concentration $(\mathrm{mg} / \mathrm{mL})$ equivalent to 0.5 absorbance glutathione. Among the tested compounds, many compounds showed significant reducing power capacity. Compounds 4, 12, 13, and 16 showed good reducing power capacity while compounds $5,8,10 b, 17 a, 17 b, 17 c, 18$, and 19 exhibited moderate reducing power capacity in comparison with the standard Glutathione. The good reducing power capacity of these compounds was due to the presence of free $\mathrm{NH}$ group in the molecules.

\section{Conclusions}

New functionalized terphenyl derivatives incorporating various heterocyclic rings are prepared by using $4,4^{\prime \prime}$-difluoro$5^{\prime}$-hydroxy-1, $1^{\prime}: 3^{\prime}, 1^{\prime \prime}$-terphenyl- $4^{\prime}$-carbohydrazide as a key intermediate derived from $4,4^{\prime}$-difluoro chalcone as a versatile synthone. All these derivatives are characterized by spectral data. All the synthesized products are screened for their in vitro antimicrobial and antioxidant properties. The majority of the tested compounds exhibited significant antimicrobial activity and some of them showed good DPPH scavenging activity. Hence this study has widened the scope of developing easy method to synthesize fluorinated functionalized terphenyl derivatives as promising antimicrobial and antioxidant agents.

\section{Experimental}

5.1. Chemistry. Melting points were taken in open capillary tubes and are uncorrected. The purity of the compounds was confirmed by thin layer chromatography using Merck silica gel $60 \mathrm{~F}_{254}$ coated aluminium plates. IR spectra were recorded on Shimadzu-FTIR Infrared spectrometer in $\mathrm{KBr}$ $\left(\nu_{\max }\right.$ in $\left.\mathrm{cm}^{-1}\right) .{ }^{1} \mathrm{HNMR}(400 \mathrm{MHz})$ spectra were recorded on a Bruker AMX 400 spectrometer, with $5 \mathrm{~mm}$ PABBO BB-1H TUBES with TMS as internal standard. LCMS were obtained using Agilent 1200 series LC and Micromass zQ spectrometer. Elemental analysis was carried out by using VARIO EL-III (Elementar Analysensysteme GmBH).

\subsection{General Procedures for the Synthesis}

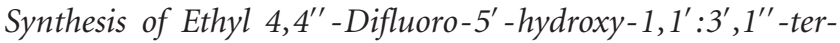
phenyl-4'-carboxylate (3). Ethyl 4,4' $4^{\prime \prime}$-difluoro- $5^{\prime}$-hydroxy$1,1^{\prime}: 3^{\prime}, 1^{\prime \prime}$-terphenyl-4' - carboxylate (3) was synthesized by the oxidative aromatization of ethyl 4,6-bis(4-fluorophenyl)2-oxocyclohex-3-ene-1-carboxylate (2) using chloramine-T, which in turn was prepared by the condensation of ethyl acetoacetate to the $4,4^{\prime}$-difluoro chalcone (1) according to the methods described in our previous work [4].

Synthesis of $4,4^{\prime \prime}$-Difluoro- $5^{\prime}$-hydroxy-1, $1^{\prime}: 3^{\prime}, 1^{\prime \prime}$-terphenyl-4' carbohydrazide (4). A mixture of ester 3 (17.7 g, $0.05 \mathrm{~mol}$ ) and hydrazine hydrate $(10 \mathrm{~mL}, 99 \%)$ in absolute ethanol $(60 \mathrm{~mL})$ was heated under reflux for $16 \mathrm{~h}$ and cooled at room temperature. The resultant solid was filtered and recrystallized from ethanol to give colorless crystals. Yield 86\%; m.p 193-196 ${ }^{\circ}$ C. IR (KBr, $\left.v \mathrm{~cm}^{-1}\right): 3290(\mathrm{NH}), 1672(\mathrm{C}=\mathrm{O}), 1219$ (C-F). ${ }^{1} \mathrm{H}$ NMR (400 MHz, DMSO-d 6 , $\delta$ ppm): 4.18 (s, 2H, $\mathrm{NH}_{2}$ ), 6.99-7.69 (m, 10H, Ar-H), 9.19 (s, 1H, NH), 9.87 (s, 1H, OH). LCMS: $m / z 341.0\left(\mathrm{M}^{+}+1\right)$. C H N Analysis; calculated for $\mathrm{C}_{19} \mathrm{H}_{14} \mathrm{~F}_{2} \mathrm{~N}_{2} \mathrm{O}_{2}$ : C, 67.05; H, 4.15; N, 8.23. Found: C, 67.02; H, 4.16; N, 8.20.

Synthesis of $\left(4,4^{\prime \prime}\right.$-Difluoro-5'-hydroxy-1, $1^{\prime}: 3^{\prime}, 1^{\prime \prime}$-terphenyl$\left.4^{\prime}-y l\right)(3,5-$ dimethyl-1H-pyrazol-1-yl)methanone (5). To a mixture of carbohydrazide $4(3.4 \mathrm{~g}, 0.01 \mathrm{~mol})$ and acetylacetone $(1.0 \mathrm{~g}, 0.01 \mathrm{~mol})$ in ethanol $(20 \mathrm{~mL})$ triethylamine $(1 \mathrm{~mL})$ was added, and the mixture was refluxed for $10 \mathrm{~h}$. The precipitated solid was filtered off and recrystallized from ethanol. Yield 52\%; m.p $166-168^{\circ} \mathrm{C}$. IR $\left(\mathrm{KBr}, v \mathrm{~cm}^{-1}\right)$ : 3327 $(\mathrm{OH}), 2926(\mathrm{C}-\mathrm{H}), 1604(\mathrm{C}=\mathrm{N}), 1215(\mathrm{C}-\mathrm{F}) .{ }^{1} \mathrm{H}$ NMR $\left(400 \mathrm{MHz}, \mathrm{DMSO}-\mathrm{d}_{6}, \delta \mathrm{ppm}\right): 2.29\left(\mathrm{~s}, 3 \mathrm{H}, \mathrm{CH}_{3}\right), 2.70(\mathrm{~s}$, $\left.3 \mathrm{H}, \mathrm{CH}_{3}\right), 6.28$ (d, 1H, CH), 6.95-7.71 (m, 10H, Ar-H), 9.70 (d, 1H, OH). LCMS: $m / z 407.0\left(\mathrm{M}^{+}+1\right)$. C H N Analysis; calculated for $\mathrm{C}_{24} \mathrm{H}_{20} \mathrm{~F}_{2} \mathrm{~N}_{2} \mathrm{O}_{2}$ : C, 71.28; H, 4.49; N, 6.93. Found: C, 71.24; H, 4.47; N, 6.91.

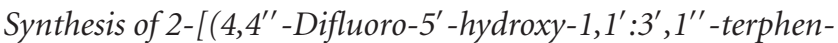
$\left.y l-4^{\prime}-y l\right)$ carbonyl]-5-methyl-2,4-dihydro-3H-pyrazol-3-one (6). To a mixture of carbohydrazide 4 (3.4 g, $0.01 \mathrm{~mol})$ and ethyl acetoacetate $(1.3 \mathrm{~g}, 0.01 \mathrm{~mol})$ in ethanol $(20 \mathrm{~mL})$ triethylamine $(1 \mathrm{~mL})$ was added, and the mixture was refluxed for $12 \mathrm{~h}$. The precipitated solid was filtered off and recrystallized from ethanol. Yield 48\%; m.p $96-98^{\circ} \mathrm{C}$. IR $\left(\mathrm{KBr}, v \mathrm{~cm}^{-1}\right)$ : $3055(\mathrm{OH}), 2985(\mathrm{C}-\mathrm{H}), 1654(\mathrm{C}=\mathrm{O})$, $1608(\mathrm{C}=\mathrm{N}), 1211(\mathrm{C}-\mathrm{F}) .{ }^{1} \mathrm{H}$ NMR (400 MHz, DMSO-d $\mathrm{d}_{6}$, $\delta$ ppm): $2.03\left(\mathrm{~s}, 2 \mathrm{H}, \mathrm{CH}_{2}\right), 2.84\left(\mathrm{~s}, 3 \mathrm{H}, \mathrm{CH}_{3}\right), 7.04-7.72(\mathrm{~m}$, 10H, Ar-H), 10.23 (s, 1H, OH). LCMS: $m / z 407.1\left(\mathrm{M}^{+}+1\right)$. $\mathrm{C} \mathrm{H} \mathrm{N}$ Analysis; calculated for $\mathrm{C}_{23} \mathrm{H}_{16} \mathrm{~F}_{2} \mathrm{~N}_{2} \mathrm{O}_{3}$ : C, 67.98; $\mathrm{H}$, 3.97; N, 6.89. Found: C, 67.94; H, 3.97; N, 6.84. 


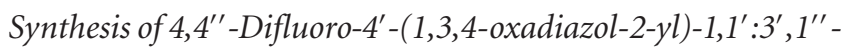
terphenyl-5'-ol (7). A mixture of carbohydrazide 4 (3.4 g, $0.01 \mathrm{~mol})$ and triethyl orthoformate $(1 \mathrm{~mL})$ in toluene $(60 \mathrm{~mL})$ was heated under reflux for $10 \mathrm{~h}$. The excess solvent was evaporated and the reaction mass was cooled to room temperature. The resultant solid was filtered and recrystallized from ethanol. Yield 58\%; m.p $193-196^{\circ} \mathrm{C}$. IR ( $\mathrm{KBr}$, $\left.v \mathrm{~cm}^{-1}\right): 3149(\mathrm{OH}), 1620(\mathrm{C}=\mathrm{N}), 1232(\mathrm{C}-\mathrm{F}) .{ }^{1} \mathrm{H}$ NMR $\left(400 \mathrm{MHz}, \mathrm{DMSO}-\mathrm{d}_{6}, \delta \mathrm{ppm} 7.12-7.79(\mathrm{~m}, 10 \mathrm{H}, \mathrm{Ar}-\mathrm{H})\right.$, $9.23(\mathrm{~s}, 1 \mathrm{H}, \mathrm{CH}), 10.57(\mathrm{~s}, 1 \mathrm{H}, \mathrm{OH})$. LCMS: $\mathrm{m} / z 351.0$ $\left(\mathrm{M}^{+}+1\right)$. C H N Analysis; calculated for $\mathrm{C}_{20} \mathrm{H}_{12} \mathrm{~F}_{2} \mathrm{~N}_{2} \mathrm{O}_{2}$ : C, 68.57; H, 3.45; N, 8.00. Found: C, 68.54; H, 3.44; N, 7.98.

Synthesis of $4^{\prime}$ - (5-Amino-1,3,4-oxadiazol-2-yl)-4,4" -difluoro$1,1^{\prime}: 3^{\prime}, 1^{\prime \prime}$-terphenyl-5'-ol (8). A mixture of carbohydrazide $4(3.4 \mathrm{~g}, 0.01 \mathrm{~mol})$ and cyanogen bromide $(1.57 \mathrm{~g}, 0.015 \mathrm{~mol})$ in absolute ethanol $(20 \mathrm{~mL})$ was refluxed for $5 \mathrm{~h}$. The reaction mass was cooled to room temperature and neutralized with saturated sodium bicarbonate solution. The precipitated solid was filtered off and recrystallized from ethanol. Yield 61\%; m.p 248-250 ${ }^{\circ}$ C. IR ( $\left.\mathrm{KBr}, v \mathrm{~cm}^{-1}\right): 3385\left(\mathrm{NH}_{2}\right), 3116$ $(\mathrm{OH}), 1600(\mathrm{C}=\mathrm{N}), 1217$ (C-F). ${ }^{1} \mathrm{H}$ NMR $(400 \mathrm{MHz}$, DMSO-d $\left.{ }_{6}, \delta \mathrm{ppm}\right): 6.93\left(\mathrm{~s}, 2 \mathrm{H}, \mathrm{NH}_{2}\right), 7.13-7.77(\mathrm{~m}, 10 \mathrm{H}$, $\mathrm{Ar}-\mathrm{H}), 10.44(\mathrm{~s}, 1 \mathrm{H}, \mathrm{OH})$. LCMS: $\mathrm{m} / z 366.0\left(\mathrm{M}^{+}+1\right)$. C H N Analysis; calculated for $\mathrm{C}_{20} \mathrm{H}_{13} \mathrm{~F}_{2} \mathrm{~N}_{3} \mathrm{O}_{2}$ : $\mathrm{C}, 65.75 ; \mathrm{H}, 3.59 ; \mathrm{N}$, 11.50. Found: C, 65.73; H, 3.57; N, 11.47 .

Synthesis of 4,4" -Difluoro-4' -(5-aryl-1,3,4-oxadiazol-2-yl)-1, $1^{\prime}: 3^{\prime}, 1^{\prime \prime}$-terphenyl-5'-ol $(9 \boldsymbol{a}-\boldsymbol{c})$. A mixture of carbohydrazide $4(3.4 \mathrm{~g}, 0.01 \mathrm{~mol})$ and aromatic carboxylic acid $(0.015 \mathrm{~mol})$ in phosphorus oxychloride $(10 \mathrm{~mL})$ was refluxed for $8 \mathrm{~h}$. The excess phosphorus oxychloride was evaporated and the cooled reaction mass was then poured into ice cold water with stirring. The separated solid was filtered and washed with saturated sodium bicarbonate solution and then with water. The product was recrystallized from ethanol-dimethyl formamide mixture.

4,4"'-Difluoro-4'-[5-(4-methoxyphenyl)-1,3,4-oxadiazol-2-yl]$1,1^{\prime}: 3^{\prime}, 1^{\prime \prime}$-terphenyl-5' -ol (9a). Yield 61\%; m.p 198-201 ${ }^{\circ} \mathrm{C}$. IR $\left(\mathrm{KBr}, v \mathrm{~cm}^{-1}\right): 3042(\mathrm{OH}), 2956(\mathrm{CH}), 1604(\mathrm{C}=\mathrm{N})$, 1220 (C-F). ${ }^{1} \mathrm{H}$ NMR (400 MHz, DMSO-d ${ }_{6}, \delta$ ppm): 3.64 (s, 3H, $\left.\mathrm{CH}_{3}\right), 6.95-7.73(\mathrm{~m}, 14 \mathrm{H}, \mathrm{Ar}-\mathrm{H}), 10.32(\mathrm{~s}, 1 \mathrm{H}, \mathrm{OH})$. LCMS: $m / z$ 457.1 $\left(\mathrm{M}^{+}+1\right)$. C H N Analysis; calculated for $\mathrm{C}_{27} \mathrm{H}_{18} \mathrm{~F}_{2} \mathrm{~N}_{2} \mathrm{O}_{3}$ : C, 71.05; H, 3.97; N, 6.14. Found: C, 71.03; $\mathrm{H}, 3.98 ; \mathrm{N}, 6.12$.

4,4'-Difluoro-4'-[5-(4-fluorophenyl)-1,3,4-oxadiazol-2-yl]-1, $1^{\prime}: 3^{\prime}, 1^{\prime \prime}$-terphenyl-5'-ol (9b). Yield 66\%; m.p $190-193^{\circ} \mathrm{C}$. IR $\left(\mathrm{KBr}, v \mathrm{~cm}^{-1}\right): 3032(\mathrm{OH}), 1604(\mathrm{C}=\mathrm{N}), 1231(\mathrm{C}-\mathrm{F}) .{ }^{1} \mathrm{H}$ NMR (400 MHz, DMSO-d 6 , $\delta$ ppm): 6.98-7.70 (m, 14H, Ar$\mathrm{H}), 9.85(\mathrm{~s}, 1 \mathrm{H}, \mathrm{OH})$. LCMS: $m / z$ 445.1 $\left(\mathrm{M}^{+}+1\right)$. C H N Analysis; calculated for $\mathrm{C}_{26} \mathrm{H}_{15} \mathrm{~F}_{3} \mathrm{~N}_{2} \mathrm{O}_{2}: \mathrm{C}, 70.27 ; \mathrm{H}, 3.40 ; \mathrm{N}$, 6.30. Found: C, $70.25 ; \mathrm{H}, 3.42 ; \mathrm{N}, 6.28$.

4,4"'-Difluoro-4' - [5-(biphenyl-4-yl)-1,3,4-oxadiazol-2-yl]-1, $1^{\prime}: 3^{\prime}, 1^{\prime \prime}$-terphenyl-5'-ol (9c). Yield 68\%; m.p $210-213^{\circ} \mathrm{C}$. IR $\left(\mathrm{KBr}, v \mathrm{~cm}^{-1}\right): 3039(\mathrm{OH}), 1606(\mathrm{C}=\mathrm{N}), 1226(\mathrm{C}-\mathrm{F}) .{ }^{1} \mathrm{H}$
NMR (400 MHz, DMSO-d 6 , $\delta$ ppm): 7.13-8.11 (m, 19H, Ar$\mathrm{H}), 10.13$ (s, 1H, OH). LCMS: $m / z 503.1\left(\mathrm{M}^{+}+1\right)$. C H N Analysis; calculated for $\mathrm{C}_{32} \mathrm{H}_{20} \mathrm{~F}_{2} \mathrm{~N}_{2} \mathrm{O}_{2}: \mathrm{C}, 76.48 ; \mathrm{H}, 4.01 ; \mathrm{N}$, 5.57. Found: C, 76.44; H, 4.02; N, 5.55.

Synthesis of Schiff Base Derivatives (10a-c). A mixture of carbohydrazide $4(3.4 \mathrm{~g}, 0.01 \mathrm{~mol})$ and aromatic aldehyde $(0.01 \mathrm{~mol})$ in absolute ethanol $(25 \mathrm{~mL})$ was heated under reflux for $4 \mathrm{~h}$ in presence of concentrated $\mathrm{HCl}(0.5 \mathrm{~mL})$. The reaction mixture was then poured into ice cold water and filtered. The pure product was recrystallized from ethanoldimethyl formamide mixture.

4, $4^{\prime \prime}$ - Difluoro- $N^{\prime}-[(Z)$-(4-methoxyphenyl)methylidene $]-5^{\prime}$ hydroxy-1,1': $3^{\prime}, 1^{\prime \prime}$-terphenyl-4'-carbohydrazide (10a). Yield $84 \%$; m.p $177-180^{\circ} \mathrm{C}$. IR (KBr, $\left.v \mathrm{~cm}^{-1}\right)$ : $3336(\mathrm{NH}), 3056$ $(\mathrm{OH}), 1637(\mathrm{C}=\mathrm{O}), 1606(\mathrm{C}=\mathrm{N}), 1232(\mathrm{C}-\mathrm{F}) .{ }^{1} \mathrm{H}$ NMR $\left(400 \mathrm{MHz}, \mathrm{DMSO}-\mathrm{d}_{6}, \delta \mathrm{ppm}\right): 3.21\left(\mathrm{~s}, 3 \mathrm{H}, \mathrm{OCH}_{3}\right), 6.93-$ $7.81(\mathrm{~m}, 14 \mathrm{H}, \mathrm{Ar}-\mathrm{H}), 8.23(\mathrm{~s}, 1 \mathrm{H}, \mathrm{CH}), 10.32(\mathrm{~s}, 1 \mathrm{H}, \mathrm{OH})$, 11.48 (s, 1H, NH). LCMS: $m / z$ 459.1 $\left(\mathrm{M}^{+}\right)$. C H N Analysis; calculated for $\mathrm{C}_{27} \mathrm{H}_{20} \mathrm{~F}_{2} \mathrm{~N}_{2} \mathrm{O}_{3}$ : C, 70.73; H, 4.40; N, 6.11. Found: C, 70.71; H, 4.39; N, 6.08 .

4,4" -Difluoro- $N^{\prime}-[(Z)$-(4-fluorophenyl)methylidene]-5'-hydroxy-1,1': $3^{\prime}, 1^{\prime \prime}$-terphenyl-4'-carbohydrazide (10b). Yield 87\%; m.p 108-110 ${ }^{\circ}$ C. IR ( $\left.\mathrm{KBr}, v \mathrm{~cm}^{-1}\right)$ : $3354(\mathrm{NH}), 3046$ $(\mathrm{OH}), 1640(\mathrm{C}=\mathrm{O}), 1600(\mathrm{C}=\mathrm{N}), 1223(\mathrm{C}-\mathrm{F}) .{ }^{1} \mathrm{H}$ NMR $\left(400 \mathrm{MHz}, \mathrm{DMSO}-\mathrm{d}_{6}, \delta \mathrm{ppm}\right): 6.91-7.78(\mathrm{~m}, 14 \mathrm{H}, \mathrm{Ar}-\mathrm{H})$, $7.93(\mathrm{~s}, 1 \mathrm{H}, \mathrm{CH}), 9.85(\mathrm{~s}, 1 \mathrm{H}, \mathrm{OH}), 10.91(\mathrm{~s}, 1 \mathrm{H}, \mathrm{NH})$. LCMS: $m / z$ 447.1 $\left(\mathrm{M}^{+}\right)$. C H N Analysis; calculated for $\mathrm{C}_{26} \mathrm{H}_{17} \mathrm{~F}_{3} \mathrm{~N}_{2} \mathrm{O}_{2}$ : C, 69.95; H, 3.84; N, 6.28. Found: C, 69.94; $\mathrm{H}, 3.86 ; \mathrm{N}, 6.24$.

4, $4^{\prime \prime}$-Difluoro- $N^{\prime}-[(Z)$ - (biphenyl-4-yl)methylidene]-5'-hydroxy-1,1': $3^{\prime}, 1^{\prime \prime}$-terphenyl-4'-carbohydrazide (10c). Yield $82 \%$; m.p 226-228 ${ }^{\circ} \mathrm{C}$. IR ( $\left.\mathrm{KBr}, v \mathrm{~cm}^{-1}\right)$ : $3329(\mathrm{NH}), 3059$ $(\mathrm{OH}), 1647(\mathrm{C}=\mathrm{O}), 1604(\mathrm{C}=\mathrm{N}), 1217(\mathrm{C}-\mathrm{F}) .{ }^{1} \mathrm{H}$ NMR (400 MHz, DMSO-d $\left.{ }_{6}, \delta \mathrm{ppm}\right): 7.07-7.97(\mathrm{~m}, 19 \mathrm{H}, \mathrm{Ar}-\mathrm{H})$, $8.15(\mathrm{~s}, 1 \mathrm{H}, \mathrm{CH}), 10.15(\mathrm{~s}, 1 \mathrm{H}, \mathrm{OH}), 11.71(\mathrm{~s}, 1 \mathrm{H}, \mathrm{NH})$. LCMS: $m / z 505.1\left(\mathrm{M}^{+}+1\right)$. C H N Analysis; calculated for $\mathrm{C}_{32} \mathrm{H}_{22} \mathrm{~F}_{2} \mathrm{~N}_{2} \mathrm{O}_{2}$ : C, 76.18; H, 4.40; N, 5.55. Found: C, 76.14; $\mathrm{H}, 4.38 ; \mathrm{N}, 5.52$.

Acetylation of Schiff Base Derivatives (11a-c). A mixture of Schiff base $(0.01 \mathrm{~mol})$ and acetic anhydride $(10 \mathrm{~mL})$ was dissolved in ethanol $(25 \mathrm{~mL})$ and the reaction mixture was refluxed for $6 \mathrm{~h}$. The reaction mixture was then concentrated and allowed to cool. The solid product obtained was filtered, washed with water, and recrystallized using ethanol-dimethyl formamide mixture.

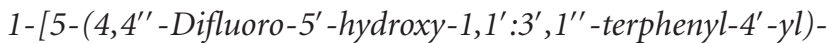
2-(4-methoxyphenyl)-1,3,4-oxadiazol-3(2H)-yl]ethanone

(11a). Yield $42 \%$; m.p $124-126^{\circ} \mathrm{C}$. IR $\left(\mathrm{KBr}, v \mathrm{~cm}^{-1}\right)$ : 3059 $(\mathrm{OH}), 2933,2837(\mathrm{CH}), 1770(\mathrm{C}=\mathrm{O}), 1608(\mathrm{C}=\mathrm{N}), 1224$ (C-F). ${ }^{1} \mathrm{H}$ NMR $\left(400 \mathrm{MHz}, \mathrm{DMSO}-\mathrm{d}_{6}, \delta \mathrm{ppm}\right): 1.84(\mathrm{~s}, 3 \mathrm{H}$, $\left.\mathrm{COCH}_{3}\right), 3.45\left(\mathrm{~s}, 3 \mathrm{H}, \mathrm{OCH}_{3}\right), 6.41(\mathrm{~s}, 1 \mathrm{H}, \mathrm{CH}), 6.74-7.85$ $(\mathrm{m}, 14 \mathrm{H}, \mathrm{Ar}-\mathrm{H}), 9.98(\mathrm{~s}, 1 \mathrm{H}, \mathrm{OH})$. LCMS: $m / z 501.1$ 
$\left(\mathrm{M}^{+}+1\right)$. C H N Analysis; calculated for $\mathrm{C}_{29} \mathrm{H}_{22} \mathrm{~F}_{2} \mathrm{~N}_{2} \mathrm{O}_{4}$ : C, 69.59; H, 4.43; N, 5.60. Found: C, 69.57; H, 4.44; N, 5.58.

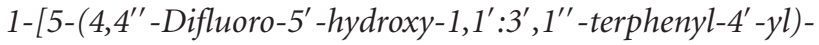
2-(4-fluorophenyl)-1,3,4-oxadiazol-3(2H)-yl]ethanone (11b). Yield $45 \%$; m.p $142-145^{\circ} \mathrm{C}$. IR $\left(\mathrm{KBr}, v \mathrm{~cm}^{-1}\right)$ : $3043(\mathrm{OH})$, $2889(\mathrm{CH}), 1762(\mathrm{C}=\mathrm{O}), 1604(\mathrm{C}=\mathrm{N}), 1232(\mathrm{C}-\mathrm{F}) .{ }^{1} \mathrm{H}$ NMR $\left(400 \mathrm{MHz}, \mathrm{DMSO}-\mathrm{d}_{6}, \delta \mathrm{ppm}\right): 1.72\left(\mathrm{~s}, 3 \mathrm{H}, \mathrm{COCH}_{3}\right), 6.52$ (s, 1H, CH), 6.82-7.91 (m, 14H, Ar-H), 9.85 (s, 1H, OH). LCMS: $m / z$ 489.1 $\left(\mathrm{M}^{+}\right)$. C H N Analysis; calculated for $\mathrm{C}_{28} \mathrm{H}_{19} \mathrm{~F}_{3} \mathrm{~N}_{2} \mathrm{O}_{3}$ : C, 68.85; H, 3.92; N, 5.74. Found: C, 68.83; $\mathrm{H}, 3.90 ; \mathrm{N}, 5.73$.

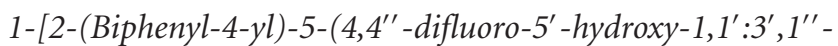
terphenyl-4'-yl)-1,3,4-oxadiazol-3(2H)-yl]ethanone (11c). Yield 50\%; m.p $133-126^{\circ} \mathrm{C}$. IR $\left(\mathrm{KBr}, v \mathrm{~cm}^{-1}\right)$ : $3032(\mathrm{OH})$, 2915 (CH), 1742 (C=O), $1611(\mathrm{C}=\mathrm{N}), 1215(\mathrm{C}-\mathrm{F}) .{ }^{1} \mathrm{H}$ NMR $\left(400 \mathrm{MHz}, \mathrm{DMSO}-\mathrm{d}_{6}, \delta \mathrm{ppm}\right): 1.94\left(\mathrm{~s}, 3 \mathrm{H}, \mathrm{COCH}_{3}\right), 6.48$ (s, 1H, CH), 7.03-7.99 (m, 19H, Ar-H), 10.15 (s, 1H, OH). LCMS: $m / z$ 547.1 $\left(\mathrm{M}^{+}\right)$. C H N Analysis; calculated for $\mathrm{C}_{34} \mathrm{H}_{24} \mathrm{~F}_{2} \mathrm{~N}_{2} \mathrm{O}_{3}$ : C, 74.71; H, 4.43; N, 5.13. Found: C, 74.72; $\mathrm{H}, 4.42 ; \mathrm{N}, 5.10$.

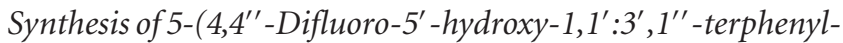
$4^{\prime}$-yl)-1,3,4-oxadiazole-2(3H)-thione (12). To a solution of carbohydrazide $4(3.4 \mathrm{~g}, 0.01 \mathrm{~mol})$ in ethanol $(10 \mathrm{~mL})$ a solution of carbon disulphide $(1.14 \mathrm{~g}, 0.015 \mathrm{~mol})$ in water $(3 \mathrm{~mL})$ and potassium hydroxide $(1 \mathrm{~g})$ was added, and the mixture was refluxed until the evolution of $\mathrm{H}_{2} \mathrm{~S}$ ceased (7$8 \mathrm{~h}$ ). The reaction mixture was allowed to cool and then acidified with dilute hydrochloric acid. The solid obtained was collected by filtration, washed with least amount of water, and recrystallized from ethanol-water $(1: 1)$. Yield $62 \%$; m.p $121-124^{\circ} \mathrm{C}$. IR (KBr, $\left.v \mathrm{~cm}^{-1}\right)$ : $3224(\mathrm{NH}), 3124$ $(\mathrm{OH}), 1604(\mathrm{C}=\mathrm{N}), 1228(\mathrm{C}-\mathrm{F}) .{ }^{1} \mathrm{H}$ NMR (400 MHz, DMSO$\left.\mathrm{d}_{6}, \delta \mathrm{ppm}\right): 7.17-7.78(\mathrm{~m}, 10 \mathrm{H}, \mathrm{Ar}-\mathrm{H}), 10.75(\mathrm{~s}, 1 \mathrm{H}, \mathrm{OH})$, 14.53 (s, 1H, NH). LCMS: $m / z 383.0\left(\mathrm{M}^{+}+1\right)$. C H N Analysis; calculated for $\mathrm{C}_{20} \mathrm{H}_{12} \mathrm{~F}_{2} \mathrm{~N}_{2} \mathrm{O}_{2} \mathrm{~S}$ : C, 62.82; $\mathrm{H}, 3.16$; N, 7.33. Found: C, 62.80; H, 3.17; N, 7.30.

Synthesis of 4-Amino-5-(4,4' -difluoro- $5^{\prime}$-hydroxy-1, $1^{\prime}: 3^{\prime}, 1^{\prime \prime}$ terphenyl-4' -yl)-2,4-dihydro-3H-1,2,4-triazole-3-thione (13). A mixture of the oxadiazole $12(3.82 \mathrm{~g}, 0.01 \mathrm{~mol})$ and hydrazine hydrate $(5 \mathrm{~mL}, 95 \%)$ in water $(20 \mathrm{~mL})$ was refluxed with stirring for $4 \mathrm{~h}$, diluted with cold water $(200 \mathrm{~mL})$, acidified by the drop wise addition of concentrated hydrochloric acid, and filtered off. The solid formed was washed with the least amount of cold water and recrystallized from ethanoldimethyl formamide mixture. Yield 58\%; m.p 240-242 ${ }^{\circ} \mathrm{C}$. IR $\left(\mathrm{KBr}, v \mathrm{~cm}^{-1}\right): 3373\left(\mathrm{NH}_{2}\right), 3259(\mathrm{NH}), 3163(\mathrm{OH}), 1616$ $(\mathrm{C}=\mathrm{N}), 1219(\mathrm{C}-\mathrm{F}) .{ }^{1} \mathrm{H}$ NMR $\left(400 \mathrm{MHz}, \mathrm{DMSO}_{-} \mathrm{d}_{6}, \delta \mathrm{ppm}\right)$ : 5.45 (s, 2H, NH $\left.\mathrm{NH}_{2}\right), 7.11-7.76(\mathrm{~m}, 10 \mathrm{H}, \mathrm{Ar}-\mathrm{H}), 10.43(\mathrm{~s}, 1 \mathrm{H}$, $\mathrm{OH}), 13.54$ (s, 1H, NH). LCMS: $m / z 397.0\left(\mathrm{M}^{+}+1\right)$. C H N Analysis; calculated for $\mathrm{C}_{20} \mathrm{H}_{14} \mathrm{~F}_{2} \mathrm{~N}_{4} \mathrm{OS}$ : C, 60.60; H, 3.56; N, 14.13. Found: C, 60.56; H, 3.57; N, 14.10.

Synthesis of Mannich Bases $(\mathbf{1 4 a - c})$. To a solution of oxadiazole 12 (3.82 g, 0.01 mole) in ethanol $(5 \mathrm{~mL})$, formaldehyde
$(0.5 \mathrm{~mL}, 37 \%)$ and secondary amine $(0.01 \mathrm{~mol})$ were added. The reaction mixture was stirred overnight. After cooling, the precipitate was filtered and recrystallized from ethanol.

5-(4, $4^{\prime \prime}$-Difluoro-5' -hydroxy-1, $1^{\prime}: 3^{\prime}, 1^{\prime \prime}$-terphenyl-4' -yl)-3(morpholin-4-ylmethyl)-1,3,4-oxadiazole-2(3H)-thione (14a). Yield 72\%; m.p 200-202 $2^{\circ}$. IR ( $\left.\mathrm{KBr}, v \mathrm{~cm}^{-1}\right): 3157(\mathrm{OH})$, 2937, $2858(\mathrm{CH}), 1620(\mathrm{C}=\mathrm{N}), 1236(\mathrm{C}-\mathrm{F}) .{ }^{1} \mathrm{H}$ NMR $\left(400 \mathrm{MHz}, \mathrm{DMSO}_{-}, \delta \mathrm{ppm}\right): 2.35\left(\mathrm{t}, 4 \mathrm{H}, \mathrm{N}-\mathrm{CH}_{2}\right.$ of morpholine), $3.45\left(\mathrm{t}, 4 \mathrm{H}, \mathrm{O}-\mathrm{CH}_{2}\right.$ of morpholine), $4.90(\mathrm{~s}$, 2H, N-CH $-\mathrm{C}$ ), 7.19-7.79 (m, 10H, Ar-H), 10.83 (s, 1H, $\mathrm{OH})$. C H N Analysis; calculated for $\mathrm{C}_{25} \mathrm{H}_{21} \mathrm{~F}_{2} \mathrm{~N}_{3} \mathrm{O}_{3} \mathrm{~S}: \mathrm{C}$, 62.36; H, 4.40; N, 8.73. Found: C, 62.34; H, 4.41; N, 8.71.

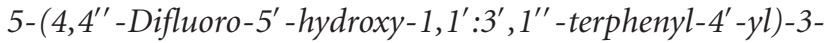
[(4-methylpiperazin-1-yl)methyl]-1,3,4-oxadiazole-2(3H)thione $(\mathbf{1 4 b})$. Yield 68\%; m.p $191-194^{\circ} \mathrm{C}$. IR $\left(\mathrm{KBr}, v \mathrm{~cm}^{-1}\right)$ : $3163(\mathrm{OH}), 2862(\mathrm{CH}), 1616(\mathrm{C}=\mathrm{N}), 1219(\mathrm{C}-\mathrm{F}) .{ }^{1} \mathrm{H}$ NMR $\left(400 \mathrm{MHz}, \mathrm{DMSO}-\mathrm{d}_{6}, \delta \mathrm{ppm}\right): 2.29\left(\mathrm{~s}, 3 \mathrm{H}, \mathrm{N}-\mathrm{CH}_{3}\right), 2.45(\mathrm{t}$, $2 \mathrm{H}, \mathrm{CH}_{2}$ ), 2.95 (t, 4H, $\mathrm{CH}_{2}$ ), 5.07 (s, 2H, N-CH $-\mathrm{N}$ ), 7.177.89 (m, 10H, Ar-H), 10.72 (s, 1H, OH). C H N Analysis; calculated for $\mathrm{C}_{26} \mathrm{H}_{24} \mathrm{~F}_{2} \mathrm{~N}_{4} \mathrm{O}_{2} \mathrm{~S}$ : C, 63.14; H, 4.89; N, 11.33. Found: C, 63.12; H, 4.87; N, 11.30.

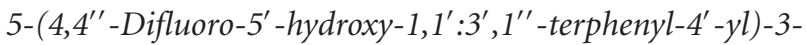
(piperidin-1-ylmethyl)-1,3,4-oxadiazole-2(3H)-thione (14c). Yield $61 \%$; m.p $98-100^{\circ} \mathrm{C}$. IR $\left(\mathrm{KBr}, v \mathrm{~cm}^{-1}\right)$ : $3173(\mathrm{OH})$, $2912(\mathrm{CH}), 1608(\mathrm{C}=\mathrm{N}), 1222(\mathrm{C}-\mathrm{F}) .{ }^{1} \mathrm{H}$ NMR $(400 \mathrm{MHz}$, DMSO- $_{6}, \delta$ ppm): 1.55-1.61 $\left(\mathrm{m}, 6 \mathrm{H}, \mathrm{CH}_{2}\right), 2.82(\mathrm{t}, 4 \mathrm{H}$, $\left.\mathrm{N}-\mathrm{CH}_{2}\right), 5.23\left(\mathrm{~s}, 2 \mathrm{H}, \mathrm{N}-\mathrm{CH}_{2}-\mathrm{N}\right), 7.11-7.76(\mathrm{~m}, 10 \mathrm{H}$, Ar-H), 10.63 (s, 1H, OH). C H N Analysis; calculated for $\mathrm{C}_{26} \mathrm{H}_{23} \mathrm{~F}_{2} \mathrm{~N}_{3} \mathrm{O}_{2} \mathrm{~S}$ : C, 65.12; H, 4.83; N, 8.76. Found: C, 65.10; $\mathrm{H}, 4.84 ; \mathrm{N}, 8.74$.

Alkylation of 1,3,4-Oxadiazole-2-thione Derivative (15a-c). Oxadiazole $12(3.82 \mathrm{~g}, 0.01 \mathrm{~mol})$ was dissolved in ethanol $(20 \mathrm{~mL})$ and $10 \%$ aqueous sodium hydroxide solution $(3.5 \mathrm{~mL})$ was added. Alkyl halide $(0.02 \mathrm{~mol})$ was added and heated to reflux for $4 \mathrm{~h}$. The reaction mixture was then poured into ice cold water, filtered, and crystallized from ethanol.

5-(Butylsulfanyl)-2-(5'-butoxy-4,4' -difluoro- $1,1^{\prime}: 3^{\prime}, 1^{\prime \prime}$-terphenyl-4'-yl)-1,3,4-oxadiazole (15a). Yield 63\%; m.p 270$272^{\circ} \mathrm{C}$. IR (KBr, $\left.v \mathrm{~cm}^{-1}\right)$ : $2964(\mathrm{CH}), 1560(\mathrm{C}=\mathrm{N}), 1170(\mathrm{C}-$ F). LCMS: $m / z 494.3\left(\mathrm{M}^{+}\right)$. C H N Analysis; calculated for $\mathrm{C}_{28} \mathrm{H}_{28} \mathrm{~F}_{2} \mathrm{~N}_{2} \mathrm{O}_{2}$ S: C, 67.99; H, 5.71; N, 5.66. Found: C, 67.96; $\mathrm{H}, 5.69$; N, 5.63 .

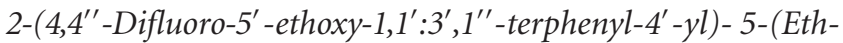
ylsulfanyl)-1,3,4-oxadiazole (15b). Yield 58\%; m.p 222$224^{\circ} \mathrm{C}$. IR (KBr, $\left.v \mathrm{~cm}^{-1}\right)$ : $2972(\mathrm{CH}), 1572(\mathrm{C}=\mathrm{N}), 1220(\mathrm{C}-$ F). LCMS: $m / z 438.4\left(\mathrm{M}^{+}\right)$. C H N Analysis; calculated for $\mathrm{C}_{24} \mathrm{H}_{20} \mathrm{~F}_{2} \mathrm{~N}_{2} \mathrm{O}_{2} \mathrm{~S}$ : C, 65.74; $\mathrm{H}, 4.60 ; \mathrm{N}, 6.39$. Found: C, 65.71; $\mathrm{H}, 4.57$; N, 6.35 . 
Ethyl ( $\left\{5-\left[4,4^{\prime \prime}\right.\right.$-difluoro-5' -(2-ethoxy-2-oxoethoxy)-1, $1^{\prime}: 3^{\prime}$, $1^{\prime \prime}$-terphenyl-4' - yl]-1,3,4-oxadiazol-2-yl\} sulfanyl)acetate

(15c). Yield 72\%; m.p $103-105^{\circ} \mathrm{C}$. IR $\left(\mathrm{KBr}, v \mathrm{~cm}^{-1}\right): 2954$ (CH), 1724 (C=O ester), $1220(\mathrm{C}-\mathrm{F}) .{ }^{1} \mathrm{H}$ NMR $(400 \mathrm{MHz}$, DMSO- $\left.\mathrm{d}_{6}, \delta \mathrm{ppm}\right): 1.14\left(\mathrm{~m}, 6 \mathrm{H}, 2 \times \mathrm{CH}_{3}\right), 3.34(\mathrm{~s}, 2 \mathrm{H}$, $\left.\mathrm{SCH}_{2}\right), 4.09\left(\mathrm{~m}, 4 \mathrm{H}, 2 \times \mathrm{CH}_{2}\right), 5.05\left(\mathrm{~s}, 2 \mathrm{H}, \mathrm{OCH}_{2}\right), 7.16-$ $7.91(\mathrm{~m}, 10 \mathrm{H}, \mathrm{Ar}-\mathrm{H})$. LCMS: $m / z$ 555.1 $\left(\mathrm{M}^{+}+1\right)$. C H N Analysis; calculated for $\mathrm{C}_{28} \mathrm{H}_{24} \mathrm{~F}_{2} \mathrm{~N}_{2} \mathrm{O}_{6} \mathrm{~S}$ : C, 60.64; $\mathrm{H}, 4.36$; N, 5.05. Found: C, 60.61; H, 4.333; N, 5.01 .

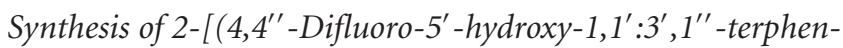
$\left.y l-4^{\prime}-y l\right)$ carbonyl]hydrazine carbothioamide (16). A mixture of carbohydrazide $4(3.4 \mathrm{~g}, 0.01 \mathrm{~mol})$, potassium thiocyanate $(0.015 \mathrm{~mol})$, and hydrochloric acid $(5 \mathrm{~mL})$ in water $(25 \mathrm{~mL})$ was refluxed for $4 \mathrm{~h}$ with stirring. The resulting solid that separated was washed with hot water and recrystallized from $75 \%$ ethanol. Yield $83 \%$; m.p $200-202^{\circ} \mathrm{C}$. IR $\left(\mathrm{KBr}, v \mathrm{~cm}^{-1}\right)$ : 3464, $3329(\mathrm{NH}), 3236(\mathrm{OH}), 1672(\mathrm{C}=\mathrm{O}), 1608(\mathrm{C}=\mathrm{N})$, 1226 (C-F). ${ }^{1} \mathrm{H}$ NMR (400 MHz, DMSO-d $\left.{ }_{6}, \delta \mathrm{ppm}\right): 6.96$, 8.19 (two singlets, $\left.2 \mathrm{H}, \mathrm{NH}_{2}\right), 7.09-7.71(\mathrm{~m}, 10 \mathrm{H}, \mathrm{Ar}-\mathrm{H})$, 9.57 (s, 1H, NH), 10.45 (s, 1H, NH), 10.75 (s, 1H, OH). LCMS: $m / z$ 400.0 $\left(\mathrm{M}^{+}+1\right)$. C H N Analysis; calculated for $\mathrm{C}_{20} \mathrm{H}_{15} \mathrm{~F}_{2} \mathrm{~N}_{3} \mathrm{O}_{2} \mathrm{~S}: \mathrm{C}, 60.14 ; \mathrm{H}, 3.79 ; \mathrm{N}, 10.52$. Found: C, 60.11; $\mathrm{H}, 3.79 ; \mathrm{N}, 10.50$.

Synthesis of 1,3-Thiazol Derivatives $(\mathbf{1 7 a}-\boldsymbol{c})$. An equimolar mixture of thiosemicarbazide derivative $16(0.399 \mathrm{~g}$, $0.001 \mathrm{~mol})$ and phenacyl bromide derivative $(0.001 \mathrm{~mol})$ in $20 \mathrm{~mL}$ of absolute ethanol was heated under reflux for $4 \mathrm{~h}$. The product that separated after cooling was collected and recrystallized from ethanol.

4,4"'-Difluoro-5'-hydroxy- $N^{\prime}$ - [4-(4-methoxyphenyl)-1,3-thiazol-2-yl]-1,1': $: 3^{\prime}, 1^{\prime \prime}$-terphenyl-4' - carbohydrazide (17a). Yield $64 \%$; m.p $213-215^{\circ} \mathrm{C}$. IR $\left(\mathrm{KBr}, v \mathrm{~cm}^{-1}\right): 3331(\mathrm{NH})$, $3196(\mathrm{OH}), 1654(\mathrm{C}=\mathrm{O}), 1606(\mathrm{C}=\mathrm{N}), 1228(\mathrm{C}-\mathrm{F}) .{ }^{1} \mathrm{H}$ NMR $\left(400 \mathrm{MHz}, \mathrm{DMSO}-\mathrm{d}_{6}, \delta \mathrm{ppm}\right): 3.75\left(\mathrm{~s}, 3 \mathrm{H}, \mathrm{OCH}_{3}\right)$, 6.91-7.73 (m, 14H, Ar-H), 9.47 (s, 1H, NH), 10.23 (s, 1H, $\mathrm{NH}), 10.37$ (s, 1H, OH). LCMS: $m / z$ 530.1 $\left(\mathrm{M}^{+}+1\right)$. C H N Analysis; calculated for $\mathrm{C}_{29} \mathrm{H}_{21} \mathrm{~F}_{2} \mathrm{~N}_{3} \mathrm{O}_{3} \mathrm{~S}$ : C, 65.77; $\mathrm{H}, 4.00$; N, 7.93. Found: C, 65.74; H, 3.96; N, 7.90.

4,4" -Difluoro-5' -hydroxy- $N^{\prime}$-[4-(4-fluorophenyl)-1,3-thiazol-2-yl]-1,1': $\mathbf{3}^{\prime}, 1^{\prime \prime}$-terphenyl-4' -carbohydrazide (17b). Yield 67\%; m.p 200-202 ${ }^{\circ}$ C. IR (KBr, $\left.v \mathrm{~cm}^{-1}\right)$ : $3329(\mathrm{NH}), 3236$ $(\mathrm{OH}), 1662(\mathrm{C}=\mathrm{O}), 1608(\mathrm{C}=\mathrm{N}), 1226(\mathrm{C}-\mathrm{F}) .{ }^{1} \mathrm{H}$ NMR $\left(400 \mathrm{MHz}, \mathrm{DMSO}-\mathrm{d}_{6}, \delta \mathrm{ppm}\right): 6.81-7.67(\mathrm{~m}, 14 \mathrm{H}, \mathrm{Ar}-\mathrm{H})$, $9.39(\mathrm{~s}, 1 \mathrm{H}, \mathrm{NH}), 10.17(\mathrm{~s}, 1 \mathrm{H}, \mathrm{NH}), 10.41(\mathrm{~s}, 1 \mathrm{H}, \mathrm{OH})$. LCMS: $m / z$ 518.2 $\left(\mathrm{M}^{+}+1\right)$. C H N Analysis; calculated for $\mathrm{C}_{28} \mathrm{H}_{18} \mathrm{~F}_{3} \mathrm{~N}_{3} \mathrm{O}_{2} \mathrm{~S}$ : C, 64.98; $\mathrm{H}, 3.51 ; \mathrm{N}$, 8.12. Found: C, 64.96; $\mathrm{H}, 3.50 ; \mathrm{N}, 8.08$.

4,4" -Difluoro-5'-hydroxy- $N^{\prime}$ - [4-(biphenyl-4-yl)-1,3-thiazol2-yl]-1, $1^{\prime}: 3^{\prime}, 1^{\prime \prime}$-terphenyl-4'-carbohydrazide (17c). Yield 60\%; m.p 169-172 ${ }^{\circ} \mathrm{C}$. IR ( $\left.\mathrm{KBr}, v \mathrm{~cm}^{-1}\right): 3324(\mathrm{NH}), 3214$ $(\mathrm{OH}), 1652(\mathrm{C}=\mathrm{O}), 1601(\mathrm{C}=\mathrm{N}), 1221(\mathrm{C}-\mathrm{F}) .{ }^{1} \mathrm{H}$ NMR (400 MHz, DMSO-d $\left.{ }_{6}, \delta \mathrm{ppm}\right): 6.69-8.14(\mathrm{~m}, 19 \mathrm{H}, \mathrm{Ar}-\mathrm{H})$, $9.41(\mathrm{~s}, 1 \mathrm{H}, \mathrm{NH}), 10.29(\mathrm{~s}, 1 \mathrm{H}, \mathrm{NH}), 10.74(\mathrm{~s}, 1 \mathrm{H}, \mathrm{OH})$.
LCMS: $m / z 576.3\left(\mathrm{M}^{+}+1\right)$. C H N Analysis; calculated for $\mathrm{C}_{34} \mathrm{H}_{23} \mathrm{~F}_{2} \mathrm{~N}_{3} \mathrm{O}_{2} \mathrm{~S}: \mathrm{C}, 70.94 ; \mathrm{H}, 4.03 ; \mathrm{N}, 7.30$. Found: $\mathrm{C}, 70.91$; $\mathrm{H}, 4.01 ; \mathrm{N}, 7.27$.

Synthesis of 4,4" -Difluoro-4' -(5-sulfanyl-4H-1,2,4-triazol-3$y$ l)-1,1': $: 3^{\prime}, 1^{\prime \prime}$-terphenyl-5'-ol (18). A suspension of the thiosemicarbazide derivative $16(0.399 \mathrm{~g}, 0.001 \mathrm{~mol})$ in sodium hydroxide solution $(10 \mathrm{~mL}, 10 \%)$ was heated under reflux for $10 \mathrm{~h}$. The reaction mixture was allowed to cool and then adjusted to $\mathrm{pH} 6$ with $10 \%$ hydrochloric acid. The precipitate that formed was filtered off, washed with water, dried, and recrystallized from ethanol. Yield 60\%; m.p 189$192^{\circ} \mathrm{C}$. IR $\left(\mathrm{KBr}, v \mathrm{~cm}^{-1}\right): 3433,3329(\mathrm{NH}), 3236(\mathrm{OH}), 2372$ (SH), $1608(\mathrm{C}=\mathrm{N}), 1228(\mathrm{C}-\mathrm{F}) .{ }^{1} \mathrm{H}$ NMR $(400 \mathrm{MHz}, \mathrm{DMSO}-$ $\left.\mathrm{d}_{6}, \delta \mathrm{ppm}\right): 4.07(\mathrm{~s}, 1 \mathrm{H}, \mathrm{SH}), 7.05-7.72(\mathrm{~m}, 10 \mathrm{H}, \mathrm{Ar}-\mathrm{H})$, 10.23 (s, $1 \mathrm{H}, \mathrm{OH}), 13.54$ (s, $1 \mathrm{H}, \mathrm{NH})$. LCMS: $\mathrm{m} / z 382.0$ $\left(\mathrm{M}^{+}+1\right)$. C H N Analysis; calculated for $\mathrm{C}_{20} \mathrm{H}_{13} \mathrm{~F}_{2} \mathrm{~N}_{3} \mathrm{OS}$ : C, $62.98 ; \mathrm{H}, 3.44 ; \mathrm{N}, 11.02$. Found: C, 62.94; H, 3.42; N, 10.99.

Synthesis of 4, $4^{\prime \prime}$-Difluoro-5' -hydroxy-N-(5-oxo-2-sulfanyl-

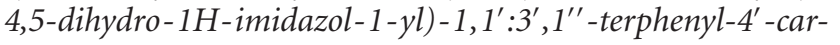
boxamide (19). A mixture of thiosemicarbazide derivative $16(0.399 \mathrm{~g}, 0.001 \mathrm{~mol})$, monochloroacetic acid $(0.001 \mathrm{~mol})$, and anhydrous sodium acetate $(0.002 \mathrm{~mol})$ in glacial acetic acid $(30 \mathrm{~mL})$ was refluxed for $8 \mathrm{~h}$. After cooling, the reaction mixture was poured on crushed ice. The resulting solid that separated was washed with hot water and recrystallized from ethanol. Yield $65 \%$; m.p $262-264^{\circ} \mathrm{C}$. IR $\left(\mathrm{KBr}, v \mathrm{~cm}^{-1}\right): 3327$ $(\mathrm{NH}), 3132(\mathrm{OH}), 1724,1635(\mathrm{C}=\mathrm{O}), 1598(\mathrm{C}=\mathrm{N}), 1222$ (C-F). ${ }^{1} \mathrm{H}$ NMR $\left(400 \mathrm{MHz}\right.$, DMSO-d $\left.{ }_{6}, \delta \mathrm{ppm}\right): 3.88(\mathrm{~s}, 2 \mathrm{H}$, $\left.\mathrm{CH}_{2}\right), 4.10(\mathrm{~s}, 1 \mathrm{H}, \mathrm{SH}), 6.94-7.78(\mathrm{~m}, 10 \mathrm{H}, \mathrm{Ar}-\mathrm{H}), 10.59(\mathrm{~s}$, $1 \mathrm{H}, \mathrm{NH}), 10.60$ (s, 1H, OH). LCMS: $m / z 440.0\left(\mathrm{M}^{+}+1\right)$. C H $\mathrm{N}$ Analysis; calculated for $\mathrm{C}_{22} \mathrm{H}_{15} \mathrm{~F}_{2} \mathrm{~N}_{3} \mathrm{O}_{3} \mathrm{~S}$ : C, $60.13 ; \mathrm{H}, 3.44$; N, 9.56. Found: C, 60.10; H, 3.42; N, 9.52.

\subsection{Biological Evaluation}

5.3.1. Antibacterial Studies. The newly synthesized compounds were screened for their antibacterial activity against Escherichia coli (ATTC-25922), Staphylococcus aureus (ATTC-25923), Pseudomonas aeruginosa (ATCC-27853), and Klebsiella pneumoniae (recultured) bacterial strains by serial plate dilution method. Serial dilutions of the drug in Mueller-Hinton broth were taken in tubes and their $\mathrm{pH}$ was adjusted to 5.0 using phosphate buffer. A standardized suspension of the test bacterium was inoculated and incubated for $16-18 \mathrm{~h}$ at $37^{\circ} \mathrm{C}$. The minimum inhibitory concentration (MIC) was noted by seeing the lowest concentration of the drug at which there was no visible growth. A number of antimicrobial discs were placed on the agar for the sole purpose of producing zones of inhibition in the bacterial lawn. About $20 \mathrm{~mL}$ of agar media was poured into each petri dish. Excess of suspension was decanted and plates were dried by placing in an incubator at $37^{\circ} \mathrm{C}$ for an hour. Using a punch, wells were made on these seeded agar plates and minimum inhibitory concentrations of the test compounds in dimethylsulfoxide (DMSO) were added into each labeled well. A control was also prepared for the 
TABLE 1: Antibacterial activity of the synthesized compounds: MIC in $\mu \mathrm{g} / \mathrm{mL}$ (Zone of inhibition in $\mathrm{mm}$ ).

\begin{tabular}{|c|c|c|c|c|}
\hline Compound & $\begin{array}{c}\text { Staphylococcus aureus } \\
\text { (ATTC-25923) }\end{array}$ & $\begin{array}{l}\text { Escherichia coli } \\
\text { (ATTC-25922) }\end{array}$ & $\begin{array}{c}\text { Pseudomonas Aeruginosa } \\
\text { (ATTC-27853) }\end{array}$ & $\begin{array}{l}\text { Klebsiella pneumonia } \\
\text { (recultured) }\end{array}$ \\
\hline 3 & $12.5(6)$ & $12.5(6)$ & $12.5(6)$ & $12.5(6)$ \\
\hline 4 & $12.5(6)$ & $12.5(8)$ & $12.5(6)$ & $12.5(6)$ \\
\hline 5 & $12.5(6)$ & $12.5(6)$ & $12.5(6)$ & $12.5(8)$ \\
\hline 6 & $12.5(6)$ & $12.5(6)$ & $12.5(6)$ & $12.5(6)$ \\
\hline 7 & $\mathrm{R}$ & $100(2)$ & $100(4)$ & $100(5)$ \\
\hline 8 & $\mathrm{R}$ & $\mathrm{R}$ & $\mathrm{R}$ & $\mathrm{R}$ \\
\hline $9 a$ & $\mathrm{R}$ & $\mathrm{R}$ & $\mathrm{R}$ & $\mathrm{R}$ \\
\hline $9 b$ & $\mathrm{R}$ & $\mathrm{R}$ & $\mathrm{R}$ & $\mathrm{R}$ \\
\hline $9 c$ & $\mathrm{R}$ & $\mathrm{R}$ & $\mathrm{R}$ & $\mathrm{R}$ \\
\hline $10 \mathrm{a}$ & $\mathrm{R}$ & $\mathrm{R}$ & $\mathrm{R}$ & $\mathrm{R}$ \\
\hline $10 \mathrm{~b}$ & $\mathrm{R}$ & $\mathrm{R}$ & $\mathrm{R}$ & $\mathrm{R}$ \\
\hline $10 \mathrm{c}$ & $\mathrm{R}$ & $\mathrm{R}$ & $\mathrm{R}$ & $\mathrm{R}$ \\
\hline $11 \mathrm{a}$ & $\mathrm{R}$ & $\mathrm{R}$ & $\mathrm{R}$ & $\mathrm{R}$ \\
\hline $11 b$ & $\mathrm{R}$ & $\mathrm{R}$ & $\mathrm{R}$ & $\mathrm{R}$ \\
\hline $11 \mathrm{c}$ & $\mathrm{R}$ & $\mathrm{R}$ & $\mathrm{R}$ & $\mathrm{R}$ \\
\hline 12 & $100(4)$ & $6.25(6)$ & $6.25(6)$ & $6.25(6)$ \\
\hline 13 & $\mathrm{R}$ & R & $\mathrm{R}$ & R \\
\hline $14 a$ & $\mathrm{R}$ & $\mathrm{R}$ & $\mathrm{R}$ & $\mathrm{R}$ \\
\hline $14 b$ & $\mathrm{R}$ & $\mathrm{R}$ & $\mathrm{R}$ & $\mathrm{R}$ \\
\hline $14 c$ & $\mathrm{R}$ & $\mathrm{R}$ & $\mathrm{R}$ & $\mathrm{R}$ \\
\hline $15 a$ & $\mathrm{R}$ & $\mathrm{R}$ & $\mathrm{R}$ & $\mathrm{R}$ \\
\hline $15 b$ & $\mathrm{R}$ & $\mathrm{R}$ & $\mathrm{R}$ & $\mathrm{R}$ \\
\hline $15 c$ & $\mathrm{R}$ & $\mathrm{R}$ & $\mathrm{R}$ & $\mathrm{R}$ \\
\hline 16 & $12.5(6)$ & $12.5(6)$ & $12.5(6)$ & $12.5(6)$ \\
\hline $17 \mathrm{a}$ & $12.5(6)$ & $12.5(6)$ & $12.5(6)$ & $12.5(6)$ \\
\hline $17 b$ & R & $12.5(6)$ & $12.5(6)$ & $12.5(6)$ \\
\hline $17 \mathrm{c}$ & $12.5(6)$ & $12.5(6)$ & $12.5(6)$ & $12.5(6)$ \\
\hline 18 & R & R & $\mathrm{R}$ & R \\
\hline 19 & $\mathrm{R}$ & $\mathrm{R}$ & $\mathrm{R}$ & $\mathrm{R}$ \\
\hline Ciprofloxacin & $6.25(20)$ & $6.25(17)$ & $6.25(19)$ & $6.25(18)$ \\
\hline
\end{tabular}

Note: $\mathrm{R}$ indicates bacterial strains are resistant to the compounds $>100 \mu \mathrm{g} / \mathrm{mL}$.

plates in the same way using solvent DMSO. The petri dishes were prepared in triplicate and maintained at $37^{\circ} \mathrm{C}$ for $3-4$ days. Antibacterial activity was determined by measuring the diameter of inhibition zone. Activity of each compound was compared with ciprofloxacin as standard. Zone of inhibition was determined for newly synthesized compounds and the results are summarized in Table 1.

5.3.2. Antifungal Studies. Newly prepared compounds were screened for their antifungal activity against Aspergillus flavus (NCIM No. 524), Aspergillus fumigates (NCIM No. 902), Penicillium (S. aureus) (recultured), and Trichophyton mentagrophytes (recultured) in DMSO by serial plate dilution method. Sabourands agar media were prepared by dissolving peptone $(1 \mathrm{~g})$, D-glucose $(4 \mathrm{~g})$ and agar $(2 \mathrm{~g})$ in distilled water $(100 \mathrm{~mL})$ and adjusting the $\mathrm{pH}$ to 5.7. Normal saline was used to make a suspension of spore of fungal strains for lawning. A loopful of particular fungal strain was transferred to $3 \mathrm{~mL}$ saline to get a suspension of corresponding species. Twenty milliliters of agar media was poured into each Petri dish. Excess of suspension were decanted and plates were dried by placing in incubator at $37^{\circ} \mathrm{C}$ for $1 \mathrm{~h}$. Using a punch, wells were made on these seeded agar plates minimum inhibitory concentrations of the test compounds in DMSO were added into each labeled well. A control was also prepared for the plates in the same way using solvent DMSO. The Petri dishes were prepared in triplicate and maintained at $37^{\circ} \mathrm{C}$ for 3-4 days. Antifungal activity was determined by measuring the diameter of inhibition zone. Activity of each compound was compared with itraconozole as standard. Zones of inhibition were determined and the results are summarized in Table 2.

5.3.3. DPPH Radical Scavenging Assay. The DPPH assay was based on the reported method [37]. Briefly, $1 \mathrm{mM}$ solution of DPPH in ethanol was prepared, and this solution $(1 \mathrm{~mL})$ was added to sample solutions $1 \mathrm{mg} / \mathrm{mL}$ of DMSO. The mixture was shaken vigorously and allowed to stand at room temperature for $20 \mathrm{~min}$. Then the absorbance was measured at $517 \mathrm{~nm}$ in a spectrophotometer. Lower absorbance of the 
TABLE 2: Antifungal activity of the synthesized compounds: MIC in $\mu \mathrm{g} / \mathrm{mL}$ (Zone of inhibition in $\mathrm{mm}$ ).

\begin{tabular}{|c|c|c|c|c|}
\hline Compound & $\begin{array}{l}\text { Penicillium marneffei } \\
\text { (recultured) }\end{array}$ & $\begin{array}{l}\text { Trichophyton mentagrophytes } \\
\text { (recultured) }\end{array}$ & $\begin{array}{l}\text { Aspergillus flavus } \\
\text { (NCIM No. 524) }\end{array}$ & $\begin{array}{l}\text { Aspergillus fumigates } \\
\text { (NCIM No. 902) }\end{array}$ \\
\hline 3 & $\mathrm{R}$ & $\mathrm{R}$ & $\mathrm{R}$ & $\mathrm{R}$ \\
\hline 4 & $\mathrm{R}$ & $\mathrm{R}$ & $\mathrm{R}$ & $\mathrm{R}$ \\
\hline 5 & $\mathrm{R}$ & $\mathrm{R}$ & $\mathrm{R}$ & $\mathrm{R}$ \\
\hline 6 & $6.25(8)$ & $6.25(8)$ & $6.25(8)$ & $6.25(8)$ \\
\hline 7 & $\mathrm{R}$ & $\mathrm{R}$ & $\mathrm{R}$ & $\mathrm{R}$ \\
\hline 8 & $6.25(8)$ & $6.25(8)$ & $6.25(8)$ & $6.25(8)$ \\
\hline $9 a$ & $100(5)$ & $12.5(6)$ & $12.5(6)$ & $\mathrm{R}$ \\
\hline $9 b$ & $\mathrm{R}$ & $\mathrm{R}$ & $\mathrm{R}$ & $\mathrm{R}$ \\
\hline $9 \mathrm{c}$ & $100(2)$ & $12.5(6)$ & $12.5(6)$ & $\mathrm{R}$ \\
\hline $10 \mathrm{a}$ & $12.5(6)$ & $\mathrm{R}$ & $\mathrm{R}$ & $\mathrm{R}$ \\
\hline $10 \mathrm{~b}$ & $6.25(8)$ & $6.25(8)$ & $6.25(8)$ & $6.25(8)$ \\
\hline $10 \mathrm{c}$ & $\mathrm{R}$ & $\mathrm{R}$ & $\mathrm{R}$ & $\mathrm{R}$ \\
\hline $11 \mathrm{a}$ & $100(4)$ & $12.5(6)$ & $12.5(6)$ & $\mathrm{R}$ \\
\hline $11 \mathrm{~b}$ & $12.5(6)$ & $12.5(6)$ & $12.5(6)$ & $\mathrm{R}$ \\
\hline $11 \mathrm{c}$ & $100(3)$ & $12.5(6)$ & $12.5(6)$ & $\mathrm{R}$ \\
\hline 12 & $\mathrm{R}$ & $\mathrm{R}$ & $\mathrm{R}$ & $\mathrm{R}$ \\
\hline 13 & $\mathrm{R}$ & $\mathrm{R}$ & $\mathrm{R}$ & $\mathrm{R}$ \\
\hline $14 a$ & $\mathrm{R}$ & $\mathrm{R}$ & $\mathrm{R}$ & $\mathrm{R}$ \\
\hline $14 \mathrm{~b}$ & $\mathrm{R}$ & $\mathrm{R}$ & $\mathrm{R}$ & $\mathrm{R}$ \\
\hline $14 \mathrm{c}$ & $\mathrm{R}$ & $\mathrm{R}$ & $\mathrm{R}$ & $\mathrm{R}$ \\
\hline $15 a$ & $\mathrm{R}$ & $\mathrm{R}$ & $\mathrm{R}$ & $\mathrm{R}$ \\
\hline $15 b$ & $\mathrm{R}$ & $\mathrm{R}$ & $\mathrm{R}$ & $\mathrm{R}$ \\
\hline $15 \mathrm{c}$ & $\mathrm{R}$ & $\mathrm{R}$ & $\mathrm{R}$ & $\mathrm{R}$ \\
\hline 16 & $\mathrm{R}$ & $\mathrm{R}$ & $\mathrm{R}$ & $\mathrm{R}$ \\
\hline $17 \mathbf{a}$ & $12.5(6)$ & $12.5(6)$ & $12.5(6)$ & $12.5(6)$ \\
\hline $17 \mathrm{~b}$ & $\mathrm{R}$ & $\mathrm{R}$ & $\mathrm{R}$ & $\mathrm{R}$ \\
\hline $17 \mathrm{c}$ & $12.5(6)$ & $\mathrm{R}$ & $\mathrm{R}$ & $\mathrm{R}$ \\
\hline 18 & $\mathrm{R}$ & $\mathrm{R}$ & $\mathrm{R}$ & $\mathrm{R}$ \\
\hline 19 & $\mathrm{R}$ & $12.5(8)$ & $12.5(6)$ & $12.5(8)$ \\
\hline Itraconozole & $6.25(17)$ & $6.25(19)$ & $6.25(18)$ & $6.25(18)$ \\
\hline
\end{tabular}

Note: $\mathrm{R}$ indicates fungal strains are resistant to the compounds $>100 \mu \mathrm{g} / \mathrm{mL}$.

reaction mixture indicated higher free radical scavenging activity. The capability to scavenge the DPPH radical was calculated using the following equation:

$$
\text { DPPH scavenging effect }(\%)=\left(A_{0}-\frac{A_{1}}{A_{0}}\right) \times 100 \text {, }
$$

where $A_{0}$ is the absorbance of the control reaction and $A_{1}$ is the absorbance in the presence of the samples or standards. Each sample was assayed at $1 \mathrm{mg} / \mathrm{mL}$ and all experiments were carried out in triplicate and the \% RSC is shown in Table 3.

5.3.4. Reducing Power Assay. The reducing power of the synthesized compounds was determined according to the method of Oyaizu [38]. Different concentrations of the samples $(100-1000 \mu \mathrm{g} / \mathrm{mL})$ in DMSO $(1 \mathrm{~mL})$ were mixed with phosphate buffer $(2.5 \mathrm{~mL}, 0.2 \mathrm{M}, \mathrm{pH}=6.6)$ and potassium ferricyanide ( $2.5 \mathrm{~mL}, 1 \%$ solution). The mixture was incubated at $50^{\circ} \mathrm{C}$ for $20 \mathrm{~min}$. after which $10 \%$ trichloroacetic acid $(2.5 \mathrm{~mL})$ was added to the mixture, which was then centrifuged for $10 \mathrm{~min}$. The upper layer of solution $(2.5 \mathrm{~mL})$ was mixed with distilled water $(2.5 \mathrm{~mL})$ and $\mathrm{FeCl}_{3}(0.5 \mathrm{~mL}, 0.1 \%)$ and then the absorbance at $700 \mathrm{~nm}$ was measured using a spectrophotometer. Higher absorbance of the reaction mixture indicated greater reducing power. All experiments were carried out in triplicate and the reducing power assay was represented by effective concentration $(\mathrm{mg} / \mathrm{mL})$ equivalent to 0.5 absorbance glutathione (Table 3 ).

\section{Acknowledgments}

The authors are thankful to the Director of IISC, Bangalore, for NMR data. B. Narayana thanks the UGC for financial assistance through BSR one time Grant for the purchase 
TABLE 3: Antioxidant activity of synthesized compounds.

\begin{tabular}{|c|c|c|}
\hline Compounds & \% DPPH scavenging & Reducing power assay \\
\hline 3 & $8.46 \pm 4.49$ & $18.18 \pm 0.46$ \\
\hline 4 & $48.40 \pm 1.12$ & $0.78 \pm 0.01$ \\
\hline 5 & $35.44 \pm 10.47$ & $6.39 \pm 1.52$ \\
\hline 6 & $28.30 \pm 1.12$ & $10.32 \pm 0.45$ \\
\hline 7 & $10.05 \pm 2.73$ & $11.90 \pm 0.01$ \\
\hline 8 & $29.09 \pm 1.42$ & $6.15 \pm 1.25$ \\
\hline $9 a$ & $69.54 \pm 4.35$ & $14.43 \pm 3.96$ \\
\hline $9 b$ & $54.94 \pm 3.14$ & $10.45 \pm 0.92$ \\
\hline $9 c$ & $42.32 \pm 5.35$ & $12.11 \pm 2.14$ \\
\hline $10 \mathrm{a}$ & $7.66 \pm 1.85$ & $10.22 \pm 0.59$ \\
\hline $10 \mathrm{~b}$ & $15.34 \pm 2.23$ & $6.99 \pm 1.16$ \\
\hline $10 \mathrm{c}$ & $19.04 \pm 5.97$ & $8.23 \pm 2.22$ \\
\hline $11 \mathrm{a}$ & $36.81 \pm 0.64$ & $63.49 \pm 11.22$ \\
\hline $11 b$ & $32.80 \pm 0.01$ & $10.82 \pm 3.31$ \\
\hline $11 \mathrm{c}$ & $47.27 \pm 3.86$ & $21.47 \pm 16.77$ \\
\hline 12 & $72.22 \pm 2.62$ & $1.49 \pm 0.11$ \\
\hline 13 & $73.63 \pm 9.00$ & $1.65 \pm 0.19$ \\
\hline $14 a$ & $61.36 \pm 9.64$ & $7.82 \pm 1.46$ \\
\hline $14 b$ & $57.26 \pm 6.42$ & $20.51 \pm 8.21$ \\
\hline $14 \mathrm{c}$ & $49.54 \pm 3.07$ & $8.77 \pm 1.18$ \\
\hline $15 \mathrm{a}$ & $1.01 \pm 0.01$ & $26.06 \pm 4.72$ \\
\hline $15 b$ & $19.99 \pm 3.29$ & $8.25 \pm 0.96$ \\
\hline $15 \mathrm{c}$ & $68.63 \pm 9.64$ & $7.67 \pm 2.23$ \\
\hline 16 & $51.58 \pm 4.11$ & $1.12 \pm 0.07$ \\
\hline $17 \mathrm{a}$ & $16.43 \pm 2.54$ & $6.18 \pm 1.87$ \\
\hline $17 b$ & $49.09 \pm 4.99$ & $4.29 \pm 0.54$ \\
\hline $17 \mathrm{c}$ & $27.50 \pm 4.49$ & $6.07 \pm 1.66$ \\
\hline 18 & $22.22 \pm 7.48$ & $2.16 \pm 0.25$ \\
\hline 19 & $15.40 \pm 2.91$ & $5.92 \pm 1.44$ \\
\hline Glutathione & $92.09 \pm 1.09$ & $0.595 \pm 0.01$ \\
\hline
\end{tabular}

of chemicals. S. Samshuddin thanks UGC for providing financial help for the research work through UGC Research Fellowship in Sciences for Meritorious Students scheme.

\section{References}

[1] M. Gill and W. Steglich, "Pigments of fungi (Macromycetes)," Fortschritte der Chemie Organischer Naturstoffe, vol. 51, pp. 1317, 1987.

[2] J. K. Liu, "Natural terphenyls: developments since 1877," Chemical Reviews, vol. 106, no. 6, pp. 2209-2223, 2006.

[3] D. Astrue, Modern Arene Chemistry, Wiley-VCH, Weinheim, Germany, 2002.

[4] S. Samshuddin, B. Narayana, and B. K. Sarojini, "Ethyl 4, 4"difluoro-5'-hydroxy-1, 1':3', 1"'-terphenyl-4' - carboxylate," Molbank, vol. 2011, no. 4, article M745, 2011.

[5] W. Wenner, "Antitubercular agents. Derivatives of pyridinecarboxylic acid hydrazides," Journal of Organic Chemistry, vol. 18, no. 10, pp. 1333-1337, 1953.

[6] S. S. Parmar and R. Kumar, "Substituted quinazolone hydrazides as possible antituberculous agents," Journal of Medicinal Chemistry, vol. 11, no. 3, pp. 635-636, 1968.
[7] R. P. Bhamaria, R. A. Bellare, and C. V. Deliwala, "In intro effect of 1-acyl-4-alkyl-(oraryl)-thiosemicarbazides 1-(5-chlorosalicylidine)-4-alkyl-(oraryl)-thiosemicarbazones and some hydrazones of 5-chlorosalicylaldehyde against pathogenic bacteria including mycobacterium tuberculosis (H37Rv)," Indian Journal of Experimental Biology, vol. 6, no. 1, pp. 62-63, 1968.

[8] E. H. Abdel-Al, M. I. Al-Ashamawi, and B. Abdel Fattah, "Synthesis and antimicrobial testing of certain oxadiazoline and triazole derivatives," Pharmazie, vol. 38, no. 12, pp. 833835, 1983.

[9] A. K. Sen Gupta, M. Garg, and U. Chandra, "Synthesis of some new Mannich bases derived from substituted benzimidazole, benzoxazol-2-one, benzoxazol-2-thione, oxadiazol-2-thiones and their biological activities," Journal of the Indian Chemical Society, vol. 56, no. 12, pp. 1230-1232, 1979.

[10] A. K. Mansour, M. M. Eid, and N. S. A. M. Khalil, "Synthesis and reactions of some new heterocyclic carbohydrazides and related compounds as potential anticancer agents," Molecules, vol. 8, no. 10, pp. 744-755, 2003.

[11] M. M. Dutta, B. N. Goswami, and J. C. S. Kataky, "Studies on biologically active heterocycles. Part I. Synthesis and antifungal activity of some new aroyl hydrazones and 2,5disubstituted-1,3,4-oxadiazoles," Journal of Heterocyclic Chemistry, vol. 23, no. 3, pp. 793-795, 1986.

[12] S. C. Sharma, "Synthesis of new fungicides. 2-(4'-Arylthiazolyl-2'-imino)-3-aryl-4-thiazolidones," Bulletin of the Chemical Society of Japan, vol. 40, no. 10, pp. 2422-2424, 1967.

[13] V. N. Choubey and H. Singh, "Synthesis of some new fungicides," Bulletin of the Chemical Society of Japan, vol. 43, no. 7, pp. 2233-2236, 1970.

[14] W. O. Foye and P. Tovivich, "N-glucopyranosyl-5-aralkylidenerhodanines: synthesis and antibacterial and antiviral activities," Journal of Pharmaceutical Sciences, vol. 66, no. 11, pp. 1607-1611, 1977.

[15] E. B. Akerblom, "Synthesis and structure- activity relationships of a series of antibacterially active 5-(5-nitro-2-furfurylidene)thiazolones, 5-(5-nitro-2-furylpropenylidene)thiazolones, and 6-(5-nitro- 2-furyl)-4H-1,3-thiazinones," Journal of Medicinal Chemistry, vol. 17, no. 6, pp. 609-615, 1974.

[16] S. Samshuddin, B. Narayana, D. N. Shetty, and R. Raghavendra, "An efficient synthesis of 2,4,6-triaryl pyridines and their biological evaluation," Der Pharma Chemica, vol. 3, no. 3, pp. 232-240, 2011.

[17] S. Samshuddin, B. Narayana, B. K. Sarojini et al., "Antimicrobial, analgesic, DPPH scavenging activities and molecular docking study of some 1,3,5-triaryl-2-pyrazolines," Medicinal Chemistry Research, vol. 21, no. 8, pp. 2012-2022, 2011.

[18] S. Samshuddin, B. Narayana, D. N. Shetty, R. Srinivasan, and B. K. Sarojini, "(2E)-3-[4-(1H-Benzimidazol-2-ylmethoxy)3-methoxyphenyl]-1-(4, 4'-difluoro-5'-methoxy-1, 1':3', $1^{\prime \prime}$ terphenyl-4'-yl)prop-2-en-1-one," Molbank, vol. 2012, no. 3, article M764, 2012.

[19] S. Samshuddin, B. Narayana, B. K. Sarojini, R. Srinivasan, Vinayachandra, and K. R. Chandrashekar, "Synthesis, characterization and biological evaluation of some pyrazoles derived from $\alpha, \beta$-dibromo 4,4'-difluoro chalcone," Der Pharma Chemica, vol. 4, no. 2, pp. 587-592, 2012.

[20] S. Samshuddin, B. Narayana, Z. Baktir, M. Akkurt, and H. S. Yathirajan, "Synthesis, characterization and crystal structure of 1-[3,5-bis(4-fluorophenyl)-4,5-dihydro-1H-pyrazol1-yl]propan-1-one," Der Pharma Chemica, vol. 3, no. 6, pp. 487-493, 2011. 
[21] S. Samshuddin, R. J. Butcher, M. Akkurt, B. Narayana, H. S. Yathirajan, and B. K. Sarojini, "1,3-Bis(4-fluorophenyl)N,N'-(propane-1,3-diylidene)dihydroxylamine," Acta Crystallographica Section E, vol. 67, no. 8, pp. o1954-o1955, 2011.

[22] H. -K. Fun, M. Hemamalini, S. Samshuddin, B. Narayana, and H. S. Yathirajan, "1-(4, 4"'Difluoro-5'-methoxy-1, 1':3', 1"terphenyl-4'-yl) ethanone," Acta Crystallographica Section E, vol. 68, no. 1, p. o163, 2010.

[23] H. K. Fun, M. Hemamalini, S. Samshuddin, B. Narayana, and B. K. Sarojini, “(E)-1-(4, 4"'-Difluoro-5'-methoxy-1, 1':3', $1^{\prime \prime}$ terphenyl-4'-yl)-3-(6-methoxynaphthalen-2-yl) prop-2-en-1one," Acta Crystallographica Section E, vol. 67, no. 12, pp. o3327-o3328, 2011.

[24] B. Narayana, K. K. V. Raj, B. V. Ashalatha, and N. S. Kumari, "Synthesis of some new 2-(6-methoxy-2-naphthyl)-5-aryl1,3,4-oxadiazoles as possible non-steroidal anti-inflammatory and analgesic agents," Archiv der Pharmazie, vol. 338, no. 8, pp. 373-377, 2005.

[25] B. Narayana, B. V. Ashalatha, K. K. Vijaya Raj, and B. K. Sarojini, "Synthesis and studies on antimicrobial, antiinflammatory and antiproliferative activities of heterocycles derived from 4-/5-/6-/7-nitro/ 5fluoro/chloro/bromoindole2-carbohydrazides," Indian Journal of Chemistry, vol. 48, no. 12, pp. 1794-1805, 2009.

[26] S. Rollas, N. Gulerman, and H. Erdeniz, "Synthesis and antimicrobial activity of some new hydrazones of 4fluorobenzoic acid hydrazide and 3-acetyl-2,5-disubstituted1,3,4-oxadiazolines," IL Farmaco, vol. 57, no. 2, pp. 171-174, 2002.

[27] A. N. Mayekar, H. S. Yathirajan, B. Narayana, B. K. Sarojini, and N. S. Kumari, "Synthesis and antimicrobial studies on new substituted 1, 3, 4-oxadiazole derivatives bearing 6-bromonaphthalene moiety," International Journal of Chemistry, vol. 2, no. 1, pp. 38-54, 2010.

[28] K. K. Vijaya Raj, B. Narayana, B. V. Ashalatha, N. Suchetha Kumari, and B. K. Sarojini, "Synthesis of some bioactive 2-bromo-5-methoxy- $N^{\prime}$-[4-(aryl)-1,3-thiazol-2-yl]benzohydrazide derivatives," European Journal of Medicinal Chemistry, vol. 42, no. 3, pp. 425-429, 2007.

[29] B. V. Ashalatha, B. Narayana, and N. S. Kumari, "Synthesis and biological activity of novel 4-/5-/6-/7-nitro-N'-(4-aryl-1,3thiazol-2-yl) $1 H$-indole-2-carbohydrazide derivatives," Phosphorus, Sulfur and Silicon and the Related Elements, vol. 181, no. 12, pp. 2785-2795, 2006.

[30] A. L. Barry, "Procedure for testing antimicrobial agents in agar media," in Antibiotics in Laboratory Medicine, V. L. Corian, Ed., Williams and Wilkins, Baltimore, Md, USA, 1991.

[31] D. James, M. Lowry, M. J. Jaqua, and S. T. Selepak, "Detailed methodology and implementation of a semi automated serial dilution micro technique for antimicrobial susceptibility testing," Applied Microbiology, vol. 20, no. 1, pp. 46-53, 1970.

[32] C. H. Fenlon and M. H. Cynamon, "Comparative in vitro activities of ciprofloxacin and other 4-quinolones against mycobacterium tuberculosis and mycobacterium intracellulare," Antimicrobial Agents and Chemotherapy, vol. 29, no. 3, pp. 386-388, 1986.

[33] B. A. Arthington-Skaggs, M. Motley, D. W. Warnock, and C. J. Morrison, "Comparative evaluation of PASCO and national committee for clinical laboratory standards M27-A broth microdilution methods for antifungal drug susceptibility testing of yeasts," Journal of Clinical Microbiology, vol. 38, no. 6, pp. 2254-2260, 2000.
[34] A. K. Tiwari, "Antioxidants: new-generation therapeutic base for treatment of polygenic disorders," Current Science, vol. 86, no. 8, pp. 1092-1102, 2004.

[35] M. Oktay, I. Gulcin, and O. I. Kufrevioglu, "Determination of in vitro antioxidant activity of fennel (Foeniculum vulgare) seed extracts," LWT-Food Science and Technology, vol. 36, no. 2, pp. 263-271, 2003.

[36] S. Chanda and R. Dave, "In vitro models for antioxidant activity evaluation and some medicinal plants possessing antioxidant properties: an overview," African Journal of Microbiology Research, vol. 3, no. 13, pp. 981-996, 2009.

[37] M. S. Blois, "Antioxidant determinations by the use of a stable free radical," Nature, vol. 181, no. 4617, pp. 1199-1200, 1958.

[38] M. Oyaizu, "Studies on products of the browning reaction. Antioxidative activities of browning reaction products prepared from glucosamine," Japanese Journal of Nutrition, vol. 44, no. 6, pp. 307-315, 1986. 


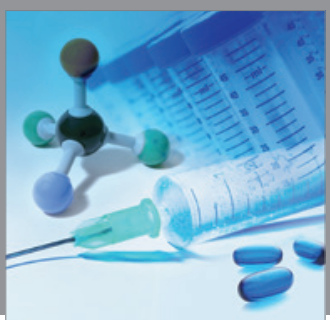

International Journal of

Medicinal Chemistry

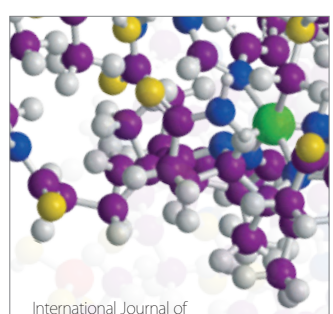

Carbohydrate Chemistry

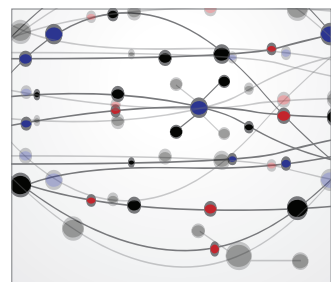

The Scientific World Journal
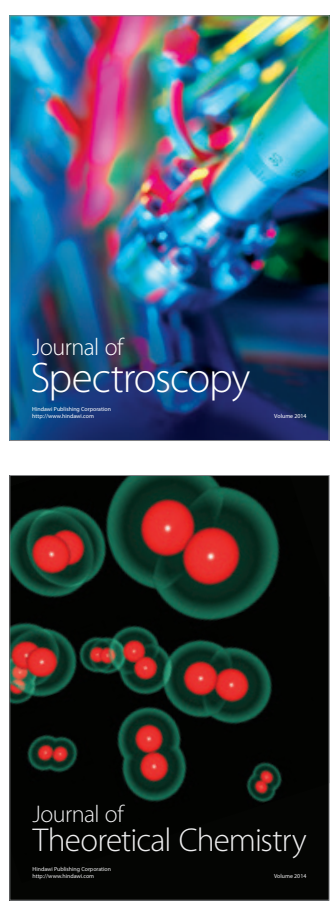
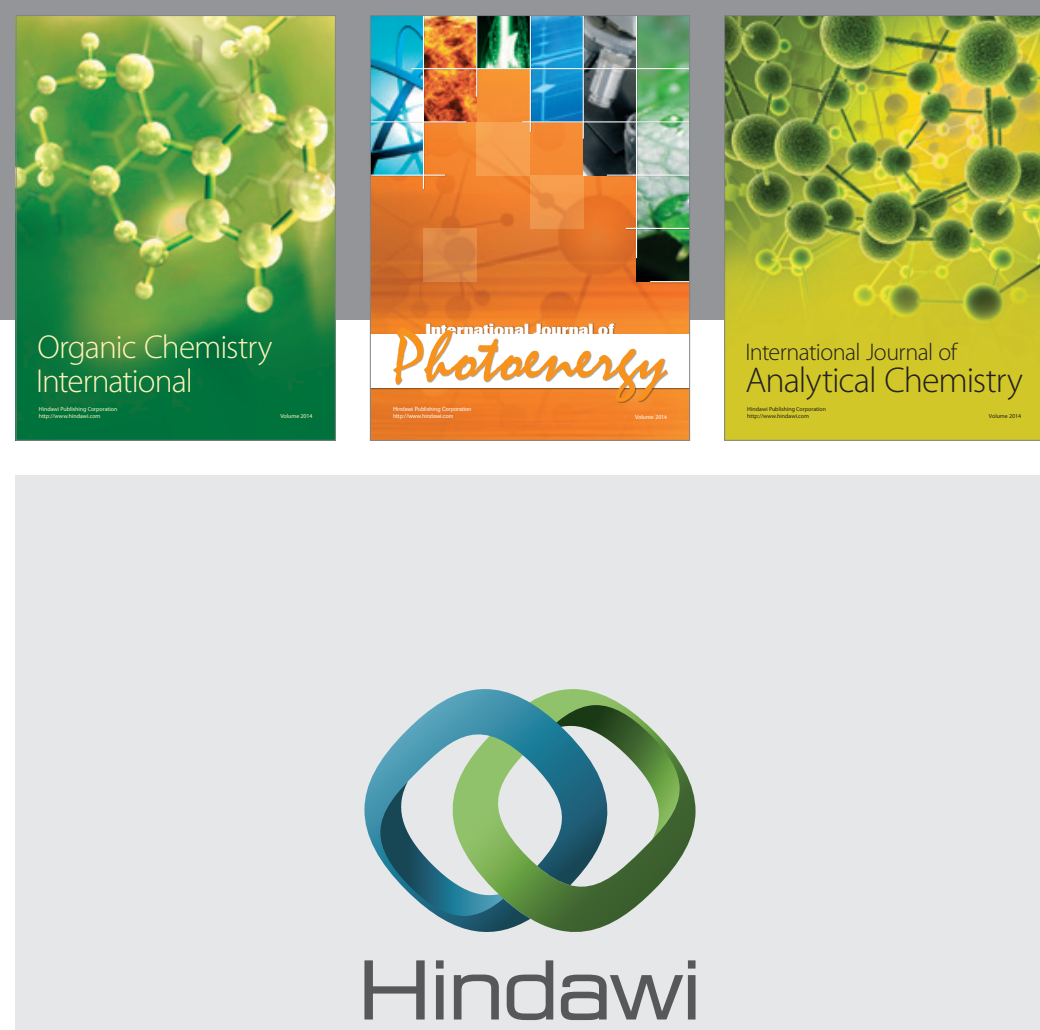

Submit your manuscripts at

http://www.hindawi.com
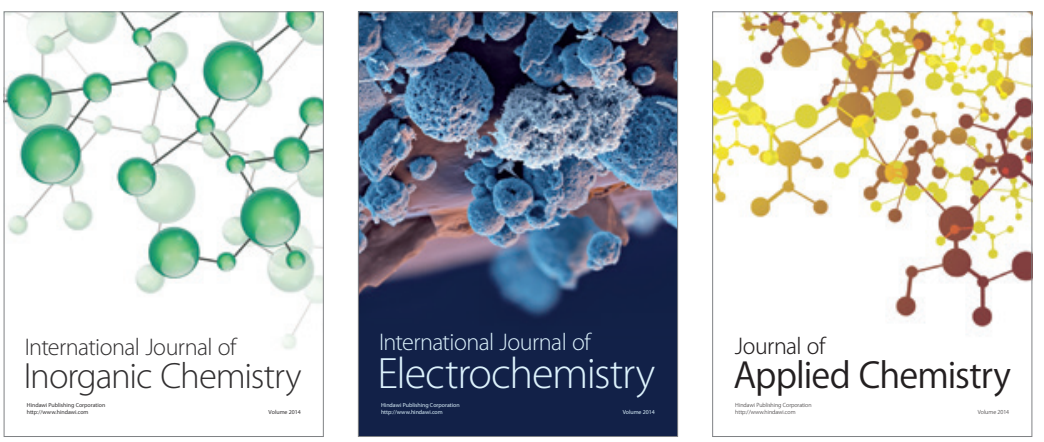

Journal of

Applied Chemistry
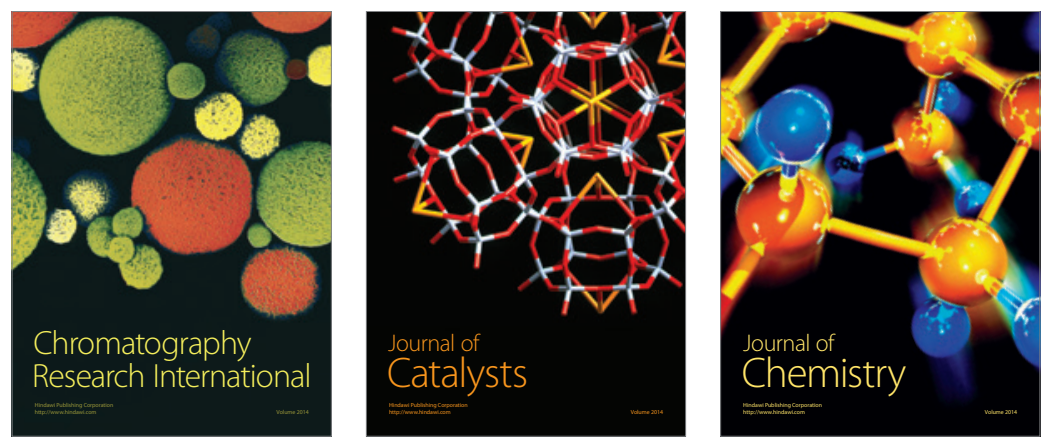
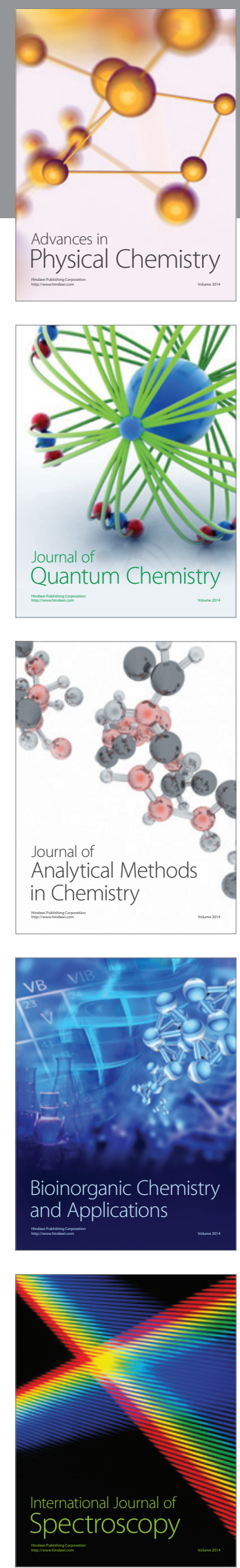